\title{
Affinity of Tau antibodies for solubilized pathological Tau species but not their immunogen or insoluble Tau aggregates predicts in vivo and ex vivo efficacy
}

Erin E. Congdon ${ }^{1 \dagger}$, Yan Lin ${ }^{1 \dagger}$, Hameetha B. Rajamohamedsait ${ }^{1}$, Dov B. Shamir ${ }^{1}$, Senthilkumar Krishnaswamy ${ }^{1}$, Wajitha J. Rajamohamedsait ${ }^{1}$, Suhail Rasool', Veronica Gonzalez', Josien Levenga 1,2, Jiaping Gu1, Charles Hoeffer ${ }^{1,2}$ and Einar M. Sigurdsson ${ }^{1,3^{*}}$

\begin{abstract}
Background: A few tau immunotherapies are now in clinical trials with several more likely to be initiated in the near future. A priori, it can be anticipated that an antibody which broadly recognizes various pathological tau aggregates with high affinity would have the ideal therapeutic properties. Tau antibodies 4E6 and 6B2, raised against the same epitope region but of varying specificity and affinity, were tested for acutely improving cognition and reducing tau pathology in transgenic tauopathy mice and neuronal cultures.

Results: Surprisingly, we here show that one antibody, 4E6, which has low affinity for most forms of tau acutely improved cognition and reduced soluble phospho-tau, whereas another antibody, 6B2, which has high affinity for various tau species was ineffective. Concurrently, we confirmed and clarified these efficacy differences in an ex vivo model of tauopathy. Alzheimer's paired helical filaments (PHF) were toxic to the neurons and increased tau levels in remaining neurons. Both toxicity and tau seeding were prevented by $4 \mathrm{E} 6$ but not by 6B2. Furthermore, 4E6 reduced PHF spreading between neurons. Interestingly, 4E6's efficacy relates to its high affinity binding to solubilized PHF, whereas the ineffective 6B2 binds mainly to aggregated PHF. Blocking 4E6's uptake into neurons prevented its protective effects if the antibody was administered after PHF had been internalized. When 4E6 and PHF were administered at the same time, the antibody was protective extracellularly.

Conclusions: Overall, these findings indicate that high antibody affinity for solubilized PHF predicts efficacy, and that acute antibody-mediated improvement in cognition relates to clearance of soluble phospho-tau. Importantly, both intra- and extracellular clearance pathways are in play. Together, these results have major implications for understanding the pathogenesis of tauopathies and for development of immunotherapies.
\end{abstract}

Keywords: Alzheimer's disease, Tau protein, Paired helical filaments, Antibodies, Immunotherapy

\footnotetext{
* Correspondence: einar.sigurdsson@nyumc.org

${ }^{\dagger}$ Equal contributors

${ }^{1}$ Departments of Neuroscience and Physiology, New York University School of Medicine, Medical Science Building, MSB459, 550 First Avenue, New York, NY 10016, USA

${ }^{3}$ Departments of Psychiatry, New York University School of Medicine, New

York, NY 10016, USA

Full list of author information is available at the end of the article
}

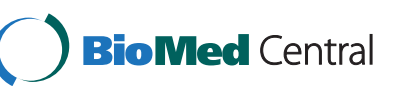

(c) 2016 The Author(s). Open Access This article is distributed under the terms of the Creative Commons Attribution 4.0 International License (http://creativecommons.org/licenses/by/4.0/), which permits unrestricted use, distribution, and reproduction in any medium, provided you give appropriate credit to the original author(s) and the source, provide a link to the Creative Commons license, and indicate if changes were made. The Creative Commons Public Domain Dedication waiver (http://creativecommons.org/publicdomain/zero/1.0/) applies to the data made available in this article, unless otherwise stated. 


\section{Background}

Tau immunotherapy was a logical approach following the success of amyloid- $\beta$ (A $\beta)$ immunotherapies in mouse models but faced resistance as tau was not thought to be accessible to antibodies. However, target engagement was feasible both intra- and extracellularly. Antibodies against tau and other targets have been detected intraneuronally [1,2], and studies over the last several decades suggested that all amyloid diseases may be transmissible between cells under proper conditions [3]. Following our initial report of active tau immunotherapy leading to clearance of tau aggregates in transgenic mice with associated functional improvements, several studies by us and others have confirmed and extended these findings (reviewed in $[4,5])$. Concurrently, spreading of tau pathology between cells in culture and via anatomically connected brain regions in animals has now been shown by several groups (reviewed in [6, 7]). A few phase I trials have now been initiated on active and passive tau immunotherapies [5]. The hope is that this approach may be more effective than targeting $A \beta$ in the later stages of the disease as tau pathology correlates better with dementia than $A \beta$ plaques [8].

Although the efficacy of tau immunotherapy has been confirmed in various models, our knowledge of the mechanisms involved is rather limited. Tau antibodies have now been detected intraneuronally in several studies by a few groups $[1,9-14]$ and such uptake shown to be necessary for acute tau clearance [10]. However, some antibodies do not appear to be taken up in appreciable amounts and are likely to primarily work extracellularly [15-17]. Such differences in uptake are well known in other immunotherapy fields and may be related to antibody charge $[18,19]$. Several tau epitopes have been successfully targeted using a similar study design (reviewed in [5]). However, very limited knowledge exists regarding the ideal affinity of antibodies and which tau species they should bind to be effective in promoting clearance of pathological tau protein. It is conceivable that very high affinity antibodies, at least against certain epitopes, may promote tau assembly or prevent their disassembly.

Most recently, we have developed a novel set of monoclonal antibodies targeting the phospho-serine 396,404 region. Two of these, 4E6 and 6B2, enter neurons and co-localize with tau [11]. In brain slice cultures, both antibodies reduce soluble phospho-tau after 6 weeks of treatment, and 4E6 has been shown to acutely reduce tau levels in primary neurons via an intracellular mechanism $[10,11]$. The two antibodies display different binding characteristics with 4E6 being phospho-selective and 6B2 having conformational properties influenced by phosphorylation and an apparent higher affinity for tau [11].
We tested the efficacy of these antibodies acutely in vivo and their ability to prevent toxicity, seeding and transmission of tau pathology in primary neuronal cultures, using paired helical filaments (PHF) isolated from an Alzheimer's brain. In addition, we examined whether neuronal uptake of antibody was necessary for efficacy, and what role timing of antibody addition had on the observed mechanism of action. Our data indicates that 4E6 acutely improves spatial learning and memory, which is associated with a reduction in soluble phospho-tau protein. Furthermore, 4E6 prevents toxicity, seeding and transmission of tau pathology even though it binds poorly to most forms of tau, whereas $6 \mathrm{~B} 2$ is ineffective although it binds strongly to most forms of tau. These unexpected results are likely to have major implications for the clinical development of tau immunotherapies, and can be explained by 4E6's high affinity for solubilized PHF, whereas the ineffective 6B2 binds primarily to aggregated but not to solubilized PHF. Hence, affinity for particular forms of tau predict efficacy. Further, whether the antibody is working outside or inside the neuron depends on the timing of PHF and antibody addition. Antibodies with access to both intra- and extracellular pools of pathological tau protein are likely to be more efficacious than antibodies acting only within one compartment.

\section{Results}

\section{In vivo studies}

4E6 acutely improves cognition in htau mice and reduces soluble phospho-tau protein, whereas 6B2 does not affect cognition or tau levels

At 11-12 months of age, the htau mice were assigned to control and treatment groups with similar cognitive (CFSM test) and motor status (Rotorod and Open Field). After baseline behavioral assessment, the mice received three antibody injections over a two week period and were re-evaluated on the same tests in addition to a fear conditioning test, followed by brain extraction for tissue analysis.

\section{Behavior}

As shown by trial errors, acute treatment with 4E6 led to significant improvements in spatial learning and memory in the CFSM test (48 \% fewer errors in posttest vs. pre-test; 13.6 (average errors) \pm 1.2 (SEM) to 7.1 $\pm 0.9, p<0.01$ ), whereas the IgG control mice did not improve compared to their pre-injection performance (Fig. 1a) These differences were not gender related. 4E6 treated males (52 \% fewer errors; $14.6 \pm 0.8$ to $7.0 \pm 1.4$, $p<0.01)$ performed similar to 4 E6 treated females $(44 \%$ fewer errors; $12.7 \pm 2.2$ to $7.1 \pm 1.2, p<0.05$ ). Repeated measures, two-way ANOVA revealed a treatment effect $(p=0.0018)$, but not a gender effect $(p=0.5145)$. 


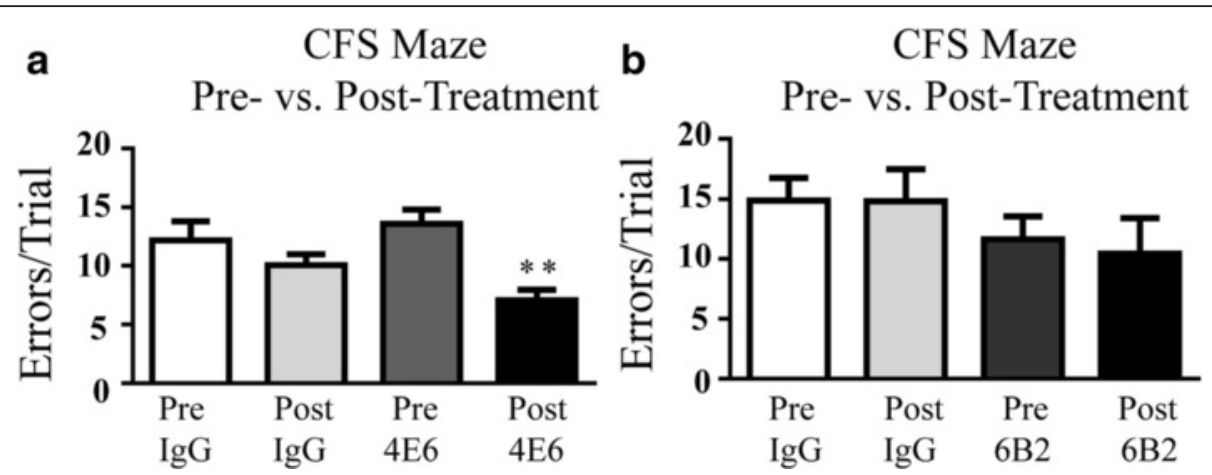

Fig. 1 Acute 4E6- but not 6B2 therapy improved spatial learning and memory in htau mice. a 4E6 immunized mice showed significant improvements in Closed Field Symmetrical (CFS) Maze (48\% fewer errors, $p<0.01$ ), compared to their pre-immunization performance, whereas control lgG treated mice did not improve. When divided by gender, both males and females treated with $4 \mathrm{E} 6$ showed significant improvement over their pre-treatment performance (52 and $44 \%$ fewer errors, $p<0.01$ and $p<0.05$, respectively, see text for average and SEM) Repeated measures, two-way ANOVA revealed a significant effect of treatment $(p=0.0018)$ but not of gender $(p=0.5145)$, indicating that the results seen are not attributable to gender differences. In contrast, animals treated with IgG did not show improvement when all animals were considered together, or when divided by gender. b 6 B2 treated mice also did not improve in the same test. ${ }^{* *}: p<0.01$

Conversely, 6B2 treatment did not result in improvements in this test (Fig. 1b). Neither antibody showed benefits in a fear conditioning test (Fig. 2a, b). Both treatment groups of mice performed similarly to IgG controls in motor function tests (rotor rod and open field tests, Fig. $2 \mathrm{c}-\mathrm{j}$ ), suggesting that the 4E6-mediated improvements in cognitive functions were direct results of the immunization, but not secondary effects from motor function changes.

\section{Immunohistochemistry, western and immunoblot analyses}

Rather mild tau pathology was detected in PHF-1 or MC1 stained brain sections (Additional file 1: Figure S1). PHF-1 stained sections had some neuropil staining but lacked cell body staining. Likewise, MC1 staining was limited, although a few intraneuronal tau aggregates were evident at high magnification. This is as expected as MC1 detects earlier tau pathology than PHF-1. There were no apparent differences between the treatment groups. Overall, we have observed a slower development, and less extensive pathology in this model compared to the initial report [20]. The observed differences in pathology may be due to several factors, such as a reduction in the transgene copy number, promoter methylation, or the cleanliness of the facility the animals are housed in. There may also be selection effects, where animals with less pathology produce larger litters and become overrepresented in the colony. Other researchers have observed a lessening of pathological severity over time, or spontaneous loss of phenotype in transgenic lines [21, 22]. Likewise, no significant differences were seen via Western blot using CP27 in either the total tau low speed supernantant, or in sarkosyl insoluble tau for either antibody (Fig. 3a-d). Tau-5, an additional total tau antibody, also showed no significant differences (Fig. 3e, f). However, acute 4E6 treatment significantly reduced soluble PHF- 1 reactive tau ( $48 \%$ reduction; $p=0.037$, Fig. 3g), whereas 6B2 did not (Fig. 3h) . This beneficial effect of the therapy was not gender related (two-way ANOVA; gender effect $(p=0.905)$, and did not appear to be oligomer specific as T22 did not reveal any differences between the 4E6 and IgG groups (Fig. 3i). Sarkosyl insoluble fractions were also probed with PHF-1 and Tau-5, with no significant differences seen between antibody and IgG treated animals for either 4E6 or 6B2. Average chemiluminescent signal for PHF-1 in animals treated with 4E6 and control IgG were $582,276 \pm 138,812$ and $491,638 \pm$ $165,340(p=0.50)$, and for Tau-5 616,980 $\pm 151,750$ and $509,180 \pm 154,157 \quad(p=0.67)$, respectively. The same analyses were performed for 6B2 and IgG treated mice with average chemiluminescent signal for PHF-1 being $327,492 \pm 92,674$ and $322,075 \pm 84,285 \quad(p=0.84)$ and for Tau-5 567,725 $\pm 179,647$ and 549,867 \pm $159,106(p=0.86)$, respectively.

For all blots prepared using low speed supernatant samples, chemiluminescent signal was normalized using GAPDH (Fig. 3). There was minor variation in some samples, but GAPDH blots showed no significant difference between the control and treated groups. Two bands are visible on the representative blot prepared from the $4 \mathrm{E} 6$ study, whereas only one band is visible in the $6 \mathrm{~B} 2$ study. This is likely due to differences in running time between blots.

\section{Mechanistic in vitro and ex vivo studies}

To further clarify the mechanisms of the positive effects of $4 \mathrm{E} 6$ and lack thereof for $6 \mathrm{~B} 2$, various in vitro and ex vivo experiments were performed. 

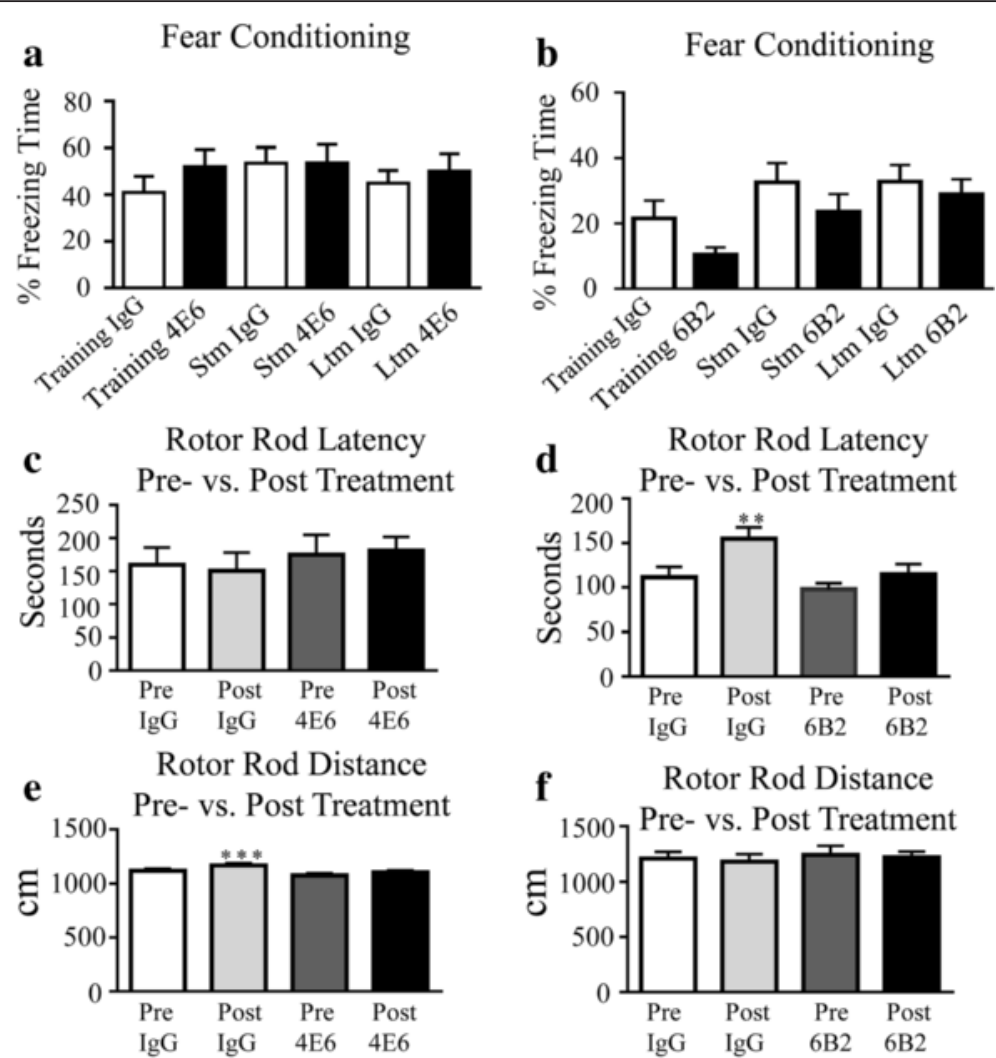

Open Field Velocity

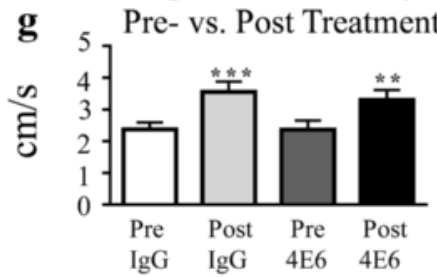

Open Field Velocity
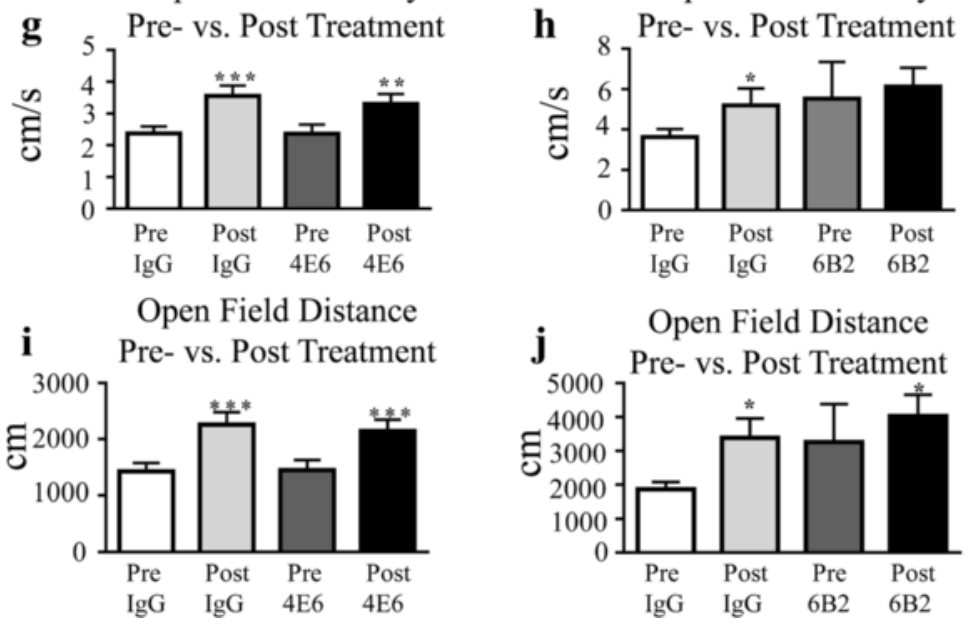

Fig. 2 Neither tau antibody led to benefits in a fear conditioning test or affected motor performance. $\mathbf{a}-\mathbf{j}$ Treatment benefits were not observed in a fear conditioning test for a $4 \mathrm{E} 6$ or b $6 \mathrm{~B} 2$, which relies on different brain circuits than the navigational test, or in motor tests $(\mathbf{c}-\mathbf{j})$, which were performed to verify that the cognitive benefits cannot be explained by confounding changes in motor performance. As expected, mice from both treated and control groups generally performed better on their post-treatment motor tests because of their pre-treatment training. ${ }^{*}: p<0.05,{ }^{* *}: p<0.01,{ }^{* * *}: p<0.001$. Stm: Short-term memory, Ltm: Long-term memory

Incubation with $A D$-derived PHF induces toxicity in primary neurons and 4E6, but not $6 B 2$, prevents these effects

Primary neuronal cultures were prepared from JNPL3 mice and exposed to human derived PHF in one of four dosing conditions (Fig. 4a, b): PHF alone, PHF added $24 \mathrm{~h}$ before antibody (PHF $\rightarrow \mathrm{Ab}$ ), PHF material and antibody added concurrently $(\mathrm{PHF}+\mathrm{Ab})$, or antibody was added $24 \mathrm{~h}$ before PHF $(\mathrm{Ab} \rightarrow \mathrm{PHF})$. PHF was used at 1 and $10 \mu \mathrm{g} / \mathrm{ml}$. Its solubility was verified at the $10 \mu \mathrm{g} / \mathrm{ml}$. No visible pellet was seen at this concentration after $100,000 \mathrm{x}$ g centrifugation for $60 \mathrm{~min}$. Prior work by others indicates that PHF can be soluble at least up to $100 \mu \mathrm{g} / \mathrm{ml}$ [23]. 


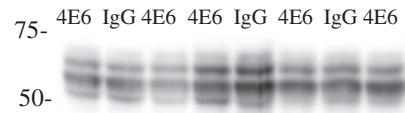

Insoluble Tau (CP27)

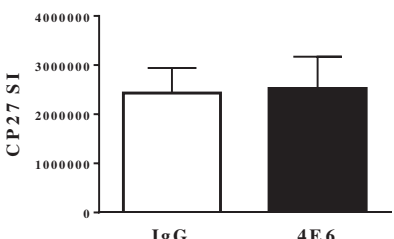

c

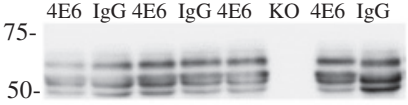

Soluble Tau (CP27)

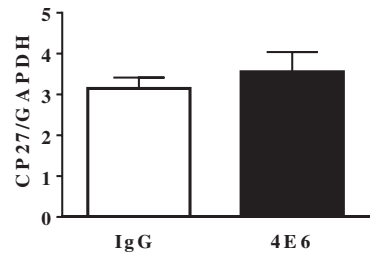

e 75 -

$50-$

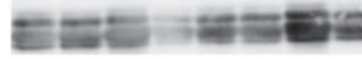

Soluble Tau (Taur5)

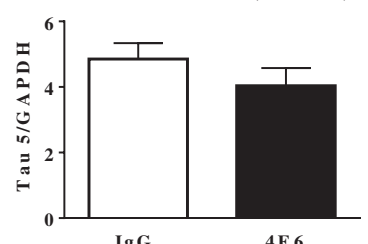

g

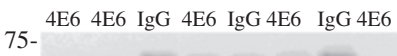

$$
50-
$$

Soluble Tau (PHF-1)

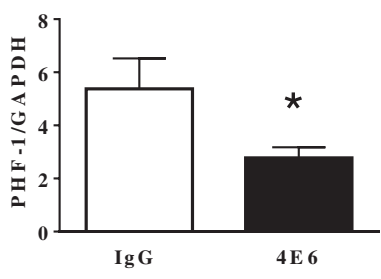

i

Anti Tau (T-22)

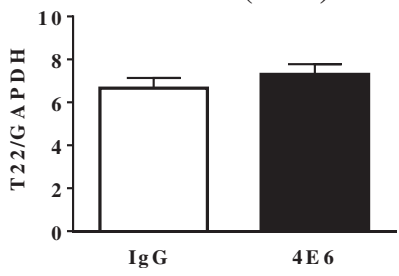

b

75- $6 \mathrm{~B} 26 \mathrm{~B} 26 \mathrm{~B} 26 \mathrm{~B} 2 \mathrm{IgG}$ IgG KO IgG

$50-$

Insoluble Tau (CP27)

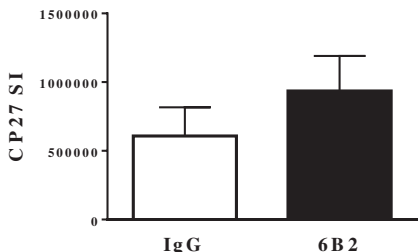

d $75-6 \mathrm{~B} 26 \mathrm{~B} 26 \mathrm{~B} 26 \mathrm{~B} 2 \mathrm{IgG} \quad \mathrm{IgG} \mathrm{KO} \quad \mathrm{IgG}$

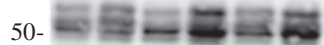

Soluble Tau (CP27)

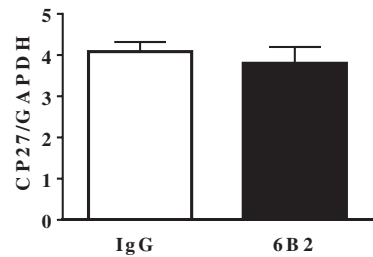

f $75-6 \mathrm{~B} 26 \mathrm{~B} 26 \mathrm{~B} 26 \mathrm{~B} 2 \mathrm{IgG} \operatorname{IgG}$ KO $\mathrm{IgG}$

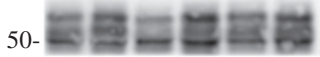

Soluble Tau (Taur5)

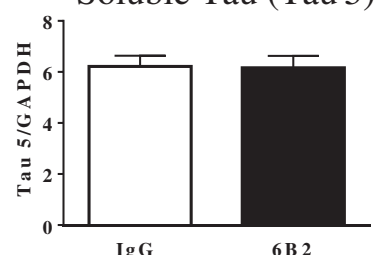

h

75- $\operatorname{IgG}$ IgG 6B2 6B2 6B2 KO IgG IgG

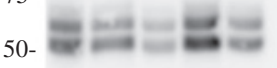

Soluble Tau (PHF-1)
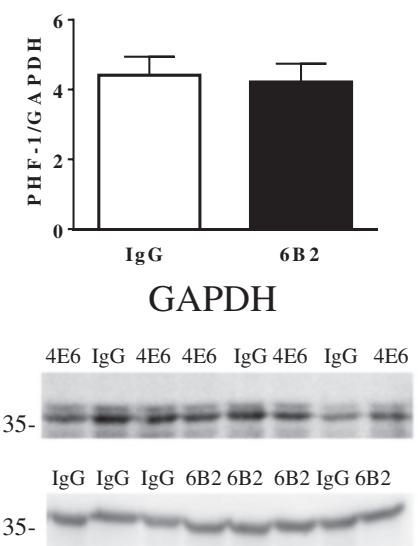

Fig. 3 (See legend on next page.) 
(See figure on previous page.)

Fig. 3 4E6, but not 6B2, reduced soluble phospho-tau levels in htau mice. a, b Insoluble tau protein (sarkosyl pellet) levels were not altered in a 4E6 or $\mathbf{b} 6$ 62 immunized mice as detected by total human tau antibody CP27, compared to lgG controls. Similar results were obtained with total tau antibody Tau-5 or phospho-tau antibody PHF-1 (not shown, see values in text). c-f Likewise, soluble tau levels (low speed supernatant; CP27, Tau-5) normalized to GAPDH were not significantly altered in 4E6 treated mice compared to lgG control group. $\mathbf{g}, \mathbf{h}$ Animals treated with 4E6 showed a significant reduction in levels of soluble PHF-1 reactive tau relative to lgG controls ( $48 \%$ reduction, $p=0.037$ ), while those treated with 6B2 showed no change. This beneficial effect of the therapy was not gender related (two-way ANOVA; gender effect: $p=0.905$ ). i Also, the cognitive benefits in the 4E6 group could not be explained by differences in T22 detected oligomeric tau as those levels did not differ between the 4E6 and IgG group (dot blot quantitation shown, similar results were seen on Western blots (not shown). *: $p<0.05$

\section{Lactate dehydrogenase assay}

Untreated control cells showed an $18 \%$ increase in LDH over 7 days, indicating that some normal cell loss occurs.

PHF $10 \mu \mathrm{g}$ Results from one-way ANOVA showed significant treatment effect $(p=0.002$; Fig. $4 d)$. Addition of $10 \mu \mathrm{g} / \mathrm{ml}$ PHF resulted in a significant increase in LDH levels relative to control samples at day 7 (a $67 \%$ increase above control, $p<0.01)$. In contrast, when $1 \mu \mathrm{g} /$ $\mathrm{ml}$ of 4E6 was added to the cultures together with the PHF material (PHF $+\mathrm{Ab}$ group) or $24 \mathrm{~h}$ after PHF $(\mathrm{PHF} \rightarrow \mathrm{Ab})$, this increase was reduced from $67 \%$ above control to 12 and $15 \%$, respectively ( $p<0.05$ relative to PHF alone). In both treatment paradigms, the groups were not significantly different from control cells. However, LDH levels in the $\mathrm{Ab} \rightarrow \mathrm{PHF}$ group were significantly higher than in the untreated cells (53\% above control, $p<0.05$ ), and did not differ significantly from PHF alone treated samples. Hence, the $\mathrm{Ab} \rightarrow \mathrm{PHF}$ treatment approach for 4E6 was ineffective, in contrast to the $\mathrm{PHF} \rightarrow \mathrm{Ab}$ and $\mathrm{PHF}+\mathrm{Ab}$ paradigms. In addition, all of the $6 \mathrm{~B} 2$ treatment groups had significantly higher LDH levels relative to untreated controls $(69,59$ and $79 \%$ above control for the $\mathrm{PHF}+\mathrm{Ab}, \mathrm{PHF} \rightarrow \mathrm{Ab}$, and $\mathrm{Ab} \rightarrow \mathrm{PHF}$ treatment groups respectively, $p<0.05)$. None of the $6 \mathrm{~B} 2$ groups differed significantly from PHF alone samples, indicating that the 6B2 antibody was ineffective under any of the paradigms in preventing PHF toxicity. IgG1 control did not influence PHF toxicity under any of the treatment conditions, again confirming the specificity of the 4E6 effect to prevent PHF toxicity.

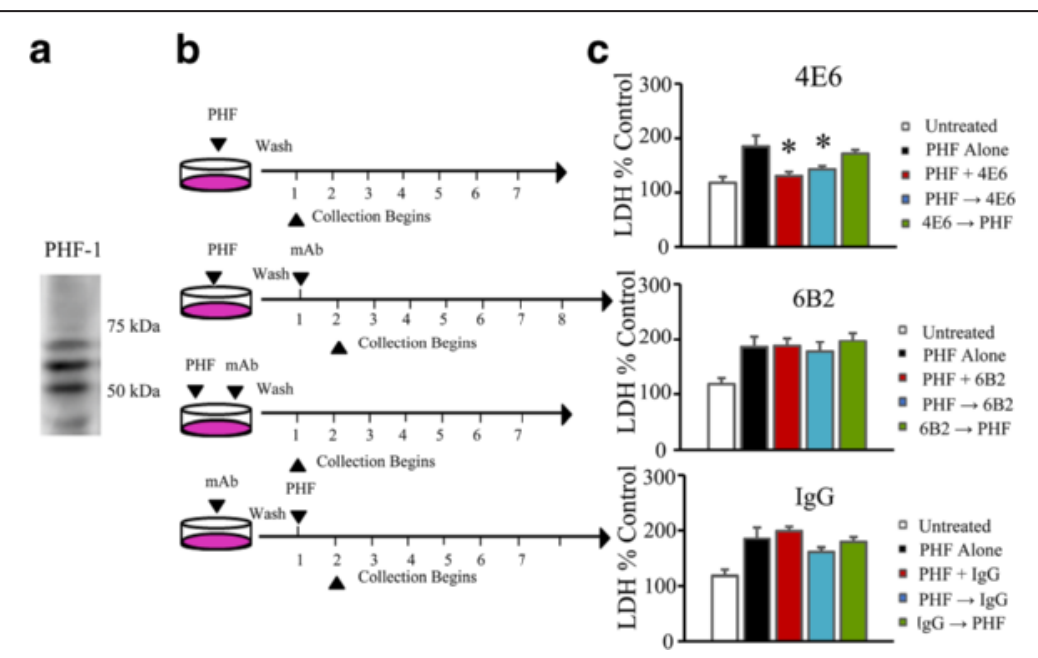

Fig. 4 PHF Characterization, dosing methods, and PHF induced toxicity as measured via LDH. a Immunoblot showing enriched human PHF tau (PHF-1 staining) derived from an Alzheimer's brain. $\mathbf{b}$ Neurons were exposed to PHF under one of four dosing paradigms. PHF was added alone, $24 \mathrm{~h}$ prior to antibody addition (PHF $\rightarrow$ Ab), together with antibody (PHF $+\mathrm{Ab}$ ), or $24 \mathrm{~h}$ after antibody (Ab $\rightarrow$ PHF). Cells were washed with Neurobasal media between each step, and collection began $24 \mathrm{~h}$ after the last treatment applied. c In cells treated with $10 \mathrm{\mu g} / \mathrm{ml}$ PHF, LDH signal averaged $67 \%$ above that of untreated controls $(p<0.01)$. 4E6 in the PHF $+\mathrm{Ab}$ and $\mathrm{PHF} \rightarrow \mathrm{Ab}$ paradigms significantly reduced $\mathrm{LDH}$ compared to $\mathrm{PHF}$ alone, and were comparable to untreated samples ( 11 and $15 \%$ above control, $p<0.05$ ), indicating that the antibody prevented toxicity. However, the Ab $\rightarrow$ PHF was not effective in reducing LDH signal (53\% above control) and showed no significant improvement over PHF alone samples. d All samples treated with $6 \mathrm{~B} 2$ showed significantly higher levels of $\mathrm{LDH}$ relative to untreated controls $(69,59$ and $79 \%$ above control for the $\mathrm{PHF}+\mathrm{Ab}, \mathrm{PHF} \rightarrow \mathrm{Ab}$, and $\mathrm{Ab} \rightarrow \mathrm{PHF}$ treatment groups respectively, $p<0.05$ ). None of the treatments with $6 \mathrm{~B} 2$ reduced $\mathrm{LDH}$ relative to PHF alone. $\mathbf{e} \operatorname{lgG}$ was also not effective in preventing the increased LDH levels triggered by the addition of PHF. LDH in the PHF $+A b, P H F \rightarrow A b$, and Ab $\rightarrow$ PHF groups was increased to 80,43 and $61 \%$ above control values $(p<0.05)$. None of the groups were significantly different from PHF alone. ${ }^{*}: p<0.05,{ }^{* *}: p<0.01,{ }^{* *}: p<0.001$ 
PHF $1 \mu \mathrm{g}$ As for $10 \mu \mathrm{g}$ PHF, a one-way ANOVA revealed significant treatment effect $(p=0.03$; data not shown). At the $1 \mu \mathrm{g} / \mathrm{ml}$ dose of PHF, LDH levels were again, as with the higher dose, significantly higher than in the untreated control cells although the toxicity was not as severe ( $35 \%$ above control, $p<0.05)$. As in the higher dose samples, 4E6 in either the $\mathrm{PHF}+\mathrm{Ab}$ or $\mathrm{PHF} \rightarrow \mathrm{Ab}$ paradigms significantly reduced LDH levels relative to PHF alone samples ( 7 and $15 \%$ below control values, $p<0.05$ for both). IgG1 control did not significantly influence PHF toxicity under any of the treatment conditions (data not shown).

\section{NeuN immunoblotting}

In addition to LDH signal, toxicity was also examined via immunoblotting with an antibody recognizing neuronal marker NeuN (Fig. 5a-c). For these and all subsequent immunoblots, untreated control cells served as internal control because typical markers could not be used due to the PHF toxicity.

PHF $10 \mu \mathrm{g}$ Results from ANOVA showed a significant effect of both treatment and time $(p<0.0001$ for both). When incubated with $10 \mu \mathrm{g} / \mathrm{ml}$ PHF, NeuN signal steadily declined and was reduced $94 \%$ relative to untreated control samples by day 7 (Fig. 5d). As was the case with the $\mathrm{LDH}$ samples, the $\mathrm{PHF}+\mathrm{Ab}$ and $\mathrm{PHF} \rightarrow \mathrm{Ab}$ paradigms for 4E6 $(1 \mu \mathrm{g} / \mathrm{ml})$ prevented PHF toxicity (samples were 16 and $24 \%$ above untreated control on day 7, Fig. 5d) and did not significantly differ from untreated control at any time point. Also as above, the $\mathrm{Ab} \rightarrow$ PHF dosing method was not effective in preventing the loss of NeuN over the treatment period $(93 \%$ loss), at each time point showed NeuN levels significantly lower than control $(p<0.0001)$ and showed no improvement over the PHF alone samples. As in the LDH assay, neither 6B2 (Fig. 5e) nor IgG1 (Fig. 5f) had any effect on PHF induced toxicity, and did not significantly differ from PHF alone.

PHF $1 \mu \mathrm{g}$ Additional groups of neurons were incubated with $1 \mu \mathrm{g} / \mathrm{ml} \mathrm{PHF}$ and $1 \mu \mathrm{g} / \mathrm{ml}$ of $4 \mathrm{E} 6$ or control IgG1 (Fig. 6) and also probed with NeuN (Fig. 6a, b). As with the higher PHF dose, the two way ANOVA results showed a significant effect of treatment and time $(p<0.0001$ and 0.007). A $30 \%$ loss of NeuN signal relative to untreated control cells was observed in the PHF alone group after seven days in culture. PHF alone samples were significantly lower than untreated controls at each time point $(p<0.01-0.001)$. As in the $10 \mu \mathrm{g}$ PHF experiments, the $\mathrm{PHF}+\mathrm{Ab}$ and $\mathrm{PHF} \rightarrow \mathrm{Ab}$ groups showed efficacy in preventing the loss of NeuN, and at day 7 were comparable to untreated controls and significantly higher that PHF alone samples $(p<0.01$ for both at day seven,

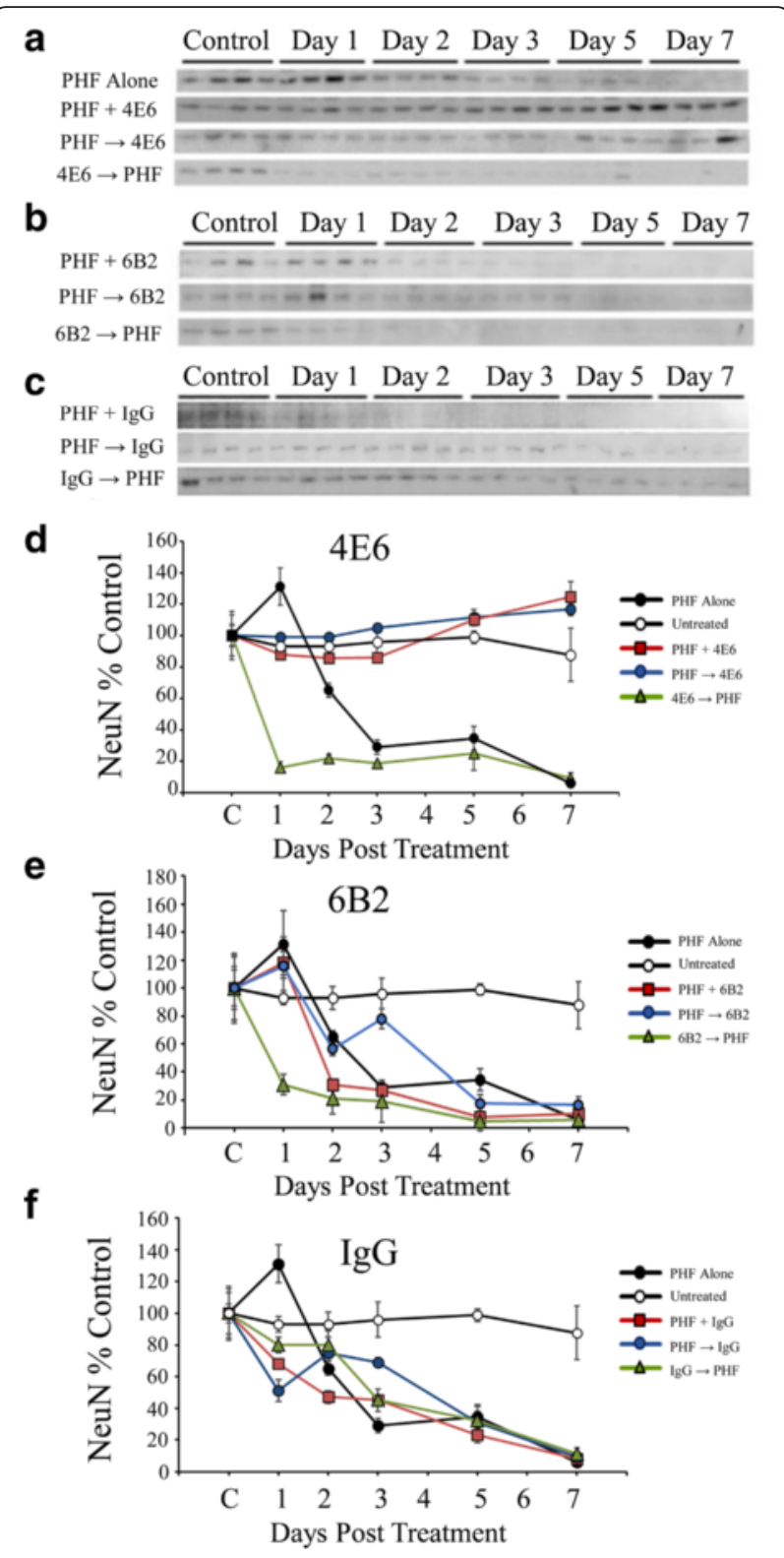

Fig. 5 4E6, but not 6B2, prevented neurotoxicity induced by $10 \mu \mathrm{g} / \mathrm{ml}$ PHF. a-c NeuN immunoblots from samples treated with $10 \mu \mathrm{g} / \mathrm{ml}$ PHF alone and with PHF and $1 \mu \mathrm{g} / \mathrm{ml}$ a 4E6, B $6 \mathrm{~B} 2$ or c control mouse IgG1. d Quantitation of signal in samples treated with PHF alone and a combination of PHF and 4E6. At $10 \mu \mathrm{g} / \mathrm{ml}$, PHF alone reduced $\mathrm{NeuN}$ levels by $94 \%$ relative to untreated control cells $(p<0.0001)$. PHF + $\mathrm{Ab}$ and $\mathrm{PHF} \rightarrow \mathrm{Ab}$ paradigms resulted in NeuN levels significantly higher than those incubated with PHF alone (116 and $124 \%$ control, $p<0.0001$ for both) and were comparable to control levels. However, the $\mathrm{Ab} \rightarrow \mathrm{PHF}$ paradigm was ineffective and showed a significantly reduced NeuN signal relative to control ( $93 \%$ loss, $p<0.0001)$ and no improvement over PHF alone samples. e 6B2 did not prevent NeuN loss under any of the dosing conditions used. None of the groups were significantly improved relative to PHF alone. $\mathbf{f}$ Similar to the 6B2 samples, the control lgG1 did not protect against the PHF induced loss of NeuN and these samples were similar to the PHF alone group 


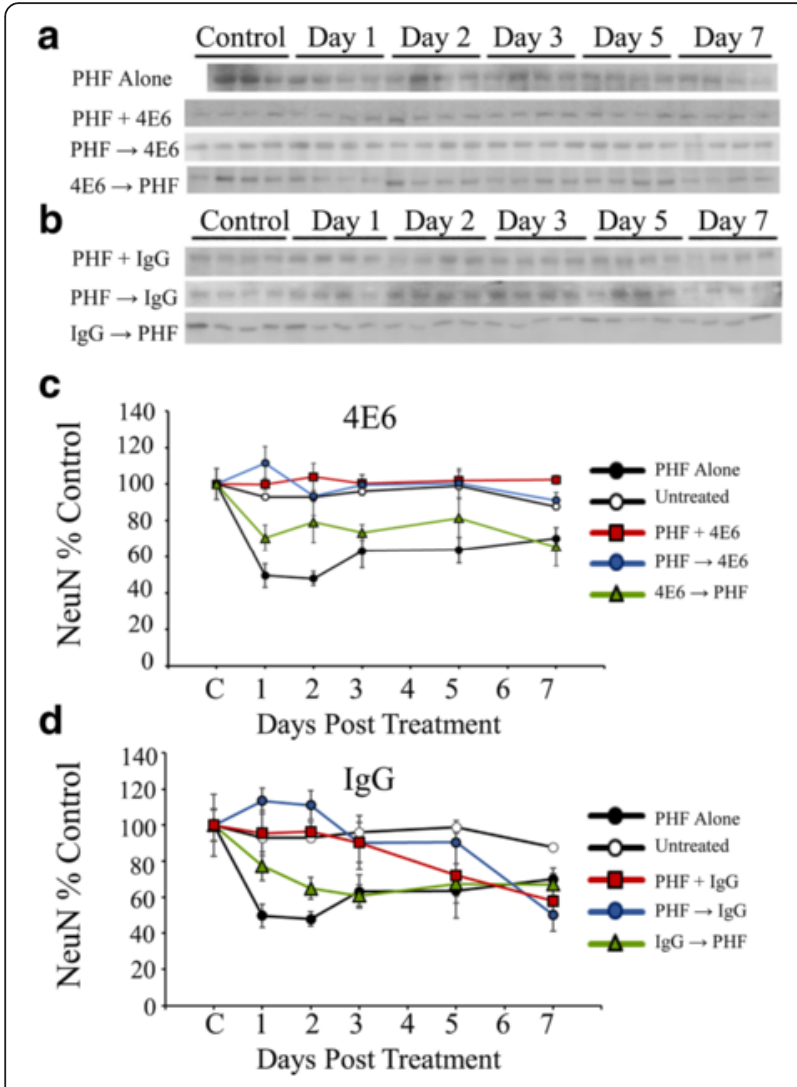

Fig. 6 Low dose PHF $(1 \mu \mathrm{g} / \mathrm{ml})$ reduced NeuN signal, and 4E6 prevented this toxicity. $\mathbf{a}, \mathbf{b}$ NeuN immunoblots for samples treated with $1 \mu \mathrm{g} / \mathrm{ml} \mathrm{PHF}$ alone or $1 \mu \mathrm{g} / \mathrm{ml} \mathrm{PHF}$ and $1 \mu \mathrm{g} / \mathrm{ml}$ a $4 \mathrm{E} 6$ or $\mathbf{b}$ control lgG1. c Chemiluminescent signal was quantified and as in the higher dose samples, exposure to PHF decreased NeuN signal (30 \% loss relative to untreated cells, $p<0.001$ ) after 7 days. For 4E6, the $\mathrm{Ab} \rightarrow$ PHF group also showed a decline in NeuN levels (36\% decrease relative to control, $p<0.05$ ) comparable to PHF alone samples. Again the $\mathrm{PHF}+\mathrm{Ab}$ and $\mathrm{PHF} \rightarrow \mathrm{Ab}$ groups had significantly higher NeuN levels at the end of the experiment (102 and $91 \%$ control values, $p<0.01$ relative to PHF alone). $\mathbf{d}$ Mouse lgG1 was ineffective in preventing NeuN loss under all of the dosing conditions and did not differ significantly from PHF alone

Fig. 6c). The $\mathrm{Ab} \rightarrow$ PHF group also showed a decline in NeuN levels ( $36 \%$ decrease over seven days relative to untreated controls, $p<0.05$ ) comparable to PHF alone samples, confirming the inefficacy of this approach. As in the higher PHF dose samples, control mouse IgG1 (Fig. 6d) was ineffective in preventing toxicity under any of the dosing conditions.

Together, the LDH and NeuN data consistently show that tau antibodies can prevent the dosedependent toxicity triggered by exposure to misfolded tau aggregates, but not all antibodies are effective. $4 \mathrm{E} 6$, but not $6 \mathrm{~B} 2$, prevented toxicity, and then only under certain conditions. When 4E6 is added prior to PHF, it is possible that the relatively low level of tau native to the neurons does not provide sufficient targets to promote the retention of antibody that is necessary to protect the cells.

In addition to both of the quantitative measures used to assess toxicity, a qualitative visual inspection of the cells was made prior to each collection. In untreated cells, cell bodies appeared healthy with an extensive network of processes. In contrast, PHF treated cells had shrunken cell bodies, retracted processes, and debris from dead cells was clearly visible under the microscope. Further, media color provides an indication of the level of cellular respiration occurring. When cultures were healthy, the changing $\mathrm{pH}$ resulted in a shift towards a more orange color. However, in cultures where cells were dying, the media remained pink indicating limited cellular activity. These PHF-induced changes were prevented in 4E6-treated cultures.

\section{E6 treatment prevents changes in tau levels caused by PHF exposure, whereas 6B2 has no effect Total Tau}

PHF $10 \mu \mathrm{g}$ In addition to measures of toxicity, the effect of PHF and antibody treatments on tau levels was also investigated via immunoblot (Fig. 7a-c). Similar to the NeuN results, a significant effect of both dosing method and time were seen $(p<0.0001$ for both). At the $10 \mu \mathrm{g} / \mathrm{ml}$ concentration, the total tau levels in the PHF alone group showed an initial decrease followed by a recovery (Fig. 7). However, by day 7 total tau levels were reduced relative to control cells (a $29 \%$ decrease, $p<0.05$ ). In contrast, the 4E6 $\mathrm{PHF}+\mathrm{Ab}$ and $\mathrm{PHF} \rightarrow \mathrm{Ab}$ groups had significantly higher tau levels (48 and $51 \%$ above control, $p<0.01$ relative to control, $p<0.001$ relative to PHF alone, Fig. 7d) than PHF alone. Again the $4 \mathrm{E} 6 \mathrm{Ab} \rightarrow$ PHF group, or any dosing paradigm of the $6 \mathrm{~B} 2$ (Fig. 7e) and IgG1 (Fig. 7f) groups were ineffective in preventing changes in tau levels at day 7 compared to PHF alone cells.

Tau/NeuN When these results were normalized using the NeuN values to take into account PHF toxicity, there were again significant effects of treatment and time by two-way ANOVA ( $p<0.0001$ for both). It became clear that the remaining cells in the PHF and 4E6 Ab $\rightarrow$ PHF groups had significantly more tau than control cells at day seven (a 5.6 and 5.5 fold increase at seven days, $p<0.0001$ for both, Fig. 7g). In the $4 \mathrm{E} 6 \mathrm{PHF}+\mathrm{Ab}$ and $\mathrm{PHF} \rightarrow \mathrm{Ab}$ groups, adjusted tau levels were comparable to untreated controls and significantly lower than the PHF alone samples $(p<0.0001$ for both). All of the 6B2 (Fig. 7h) and IgG1 (Fig. 7i) dosing groups did not differ significantly from PHF alone, and were significantly higher than untreated cells by day $7(p<0.05-0.0001)$. 
a

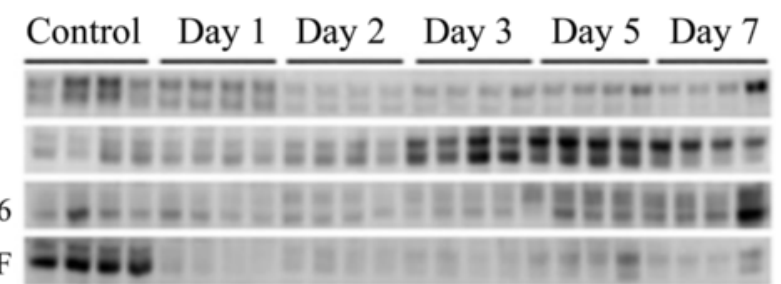

b

Control Day 1 Day 2 Day 3 Day 5 Day 7

$\mathrm{PHF}+6 \mathrm{~B} 2$

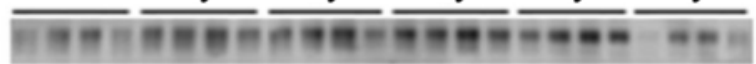

$\mathrm{PHF} \rightarrow 6 \mathrm{~B} 2$ 불

$6 \mathrm{~B} 2 \rightarrow \mathrm{PHF}$

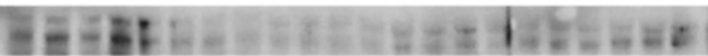

C

Control Day 1 Day 2 Day 3 Day 5 Day 7
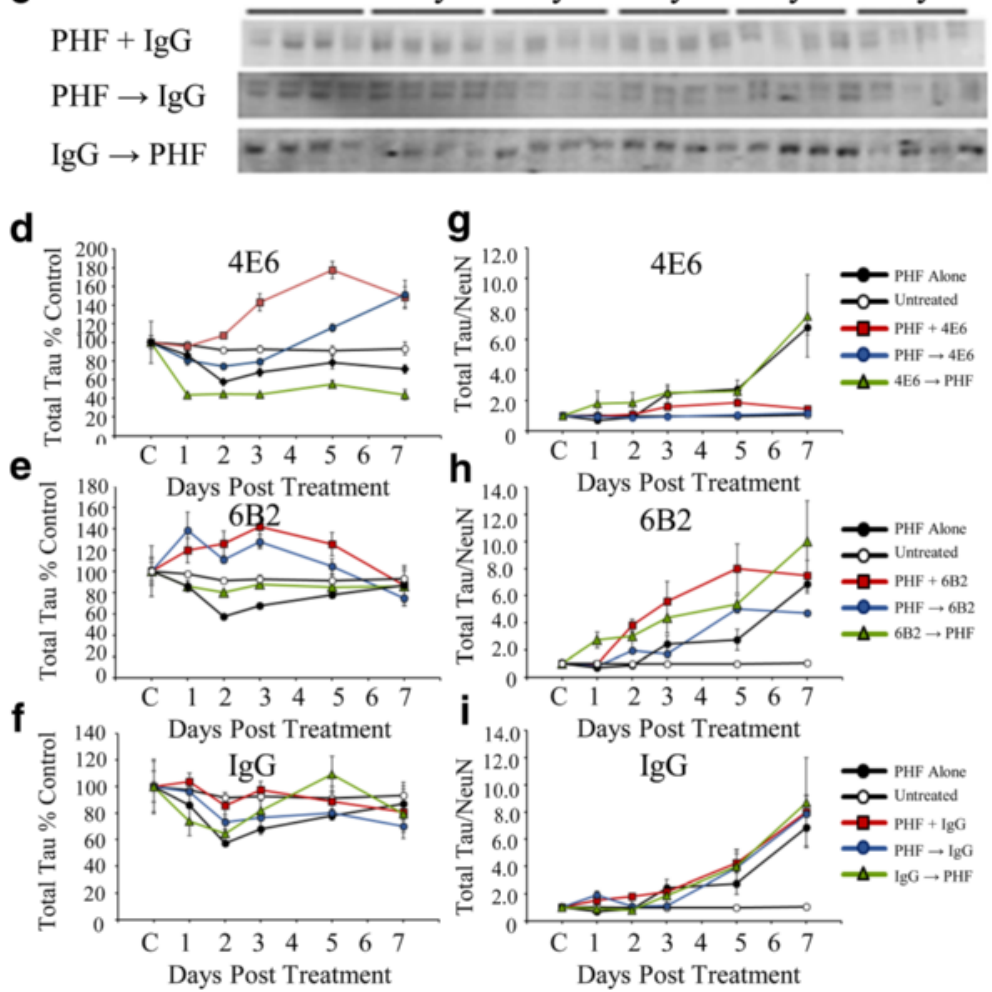

Fig. 7 4E6, but not 6B2, prevented increase in the tau/NeuN ratio caused by exposure to $10 \mu \mathrm{g} / \mathrm{ml}$ PHF. a-c Immunoblots probed with a pan-tau antibody of samples incubated with PHF or PHF in combination with $\mathbf{a} 4 \mathrm{E} 6, \mathbf{b} 6 \mathrm{~B} 2$ or c control lgG1. d Quantitation of total tau levels in samples incubated with PHF and 4E6. With PHF alone, total tau levels decreased relative to control before recovering (29\% decrease at day 7). In contrast, samples in the PHF $+\mathrm{Ab}$ and $\mathrm{PHF} \rightarrow \mathrm{Ab}$ groups had significantly higher tau levels (48 and $51 \%$ above control, $p<0.001$ relative to PHF alone). Ab $\rightarrow$ PHF samples did not significantly differ from PHF alone. e After 7 days in culture, samples treated with PHF and 6B2 were not significantly different than the PHF alone samples. $\mathbf{f}$ The lgG1 treated cells also did not differ significantly from PHF alone. $\mathbf{g}$ We then used the values obtained from the NeuN data to normalize tau levels. Due to the substantial toxicity seen using LDH and NeuN immunoblotting, tau levels alone did not provide an accurate picture of the effects of PHF exposure. Incorporating NeuN data allowed us to account for neuronal loss when assessing changes induced by PHF. Using this method, it became evident that the remaining cells in the PHF group had significantly more tau (tau/NeuN) that control cells (5.6 fold increase, $p<0.0001$ ). In the $\mathrm{PHF}+\mathrm{Ab}$ and $\mathrm{PHF} \rightarrow \mathrm{Ab}$ groups, adjusted tau levels were comparable to control and significantly lower than the PHF alone samples $(p<0.0001)$. $\mathbf{h}$ When NeuN levels were controlled for, all 6B2 treated groups had tau levels significantly higher that controls with no significant difference from PHF alone group $(p<0.05-0.0001)$. i Controlling for NeuN did not alter the pattern of results seen in cells incubated with lgG

PHF $1 \mu \mathrm{g}$ At the $1 \mu \mathrm{g} / \mathrm{ml}$ dose, the increased tau levels were evident even before normalization with NeuN level (Fig. 8a, b). Again a two-way ANOVA showed significant dosing and time effects $(p<0.0001$ for both, Fig. 8c).
PHF promoted significant increases in intracellular tau (95\% above control, $p<0.0001$ ) by experimental day 7 . The $\mathrm{PHF}+\mathrm{Ab}$ and $\mathrm{PHF} \rightarrow \mathrm{Ab}$ were not changed relative to untreated control and were significantly lower than 
a

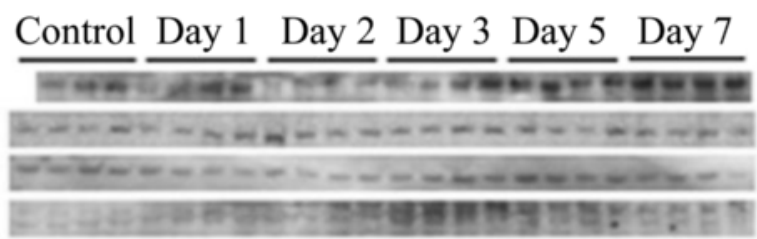

b

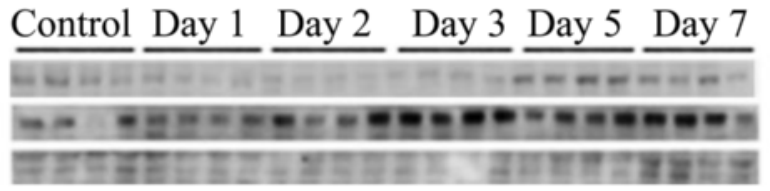

$\mathrm{IgG} \rightarrow \mathrm{PHF}$

c

$\mathbf{e}$

d
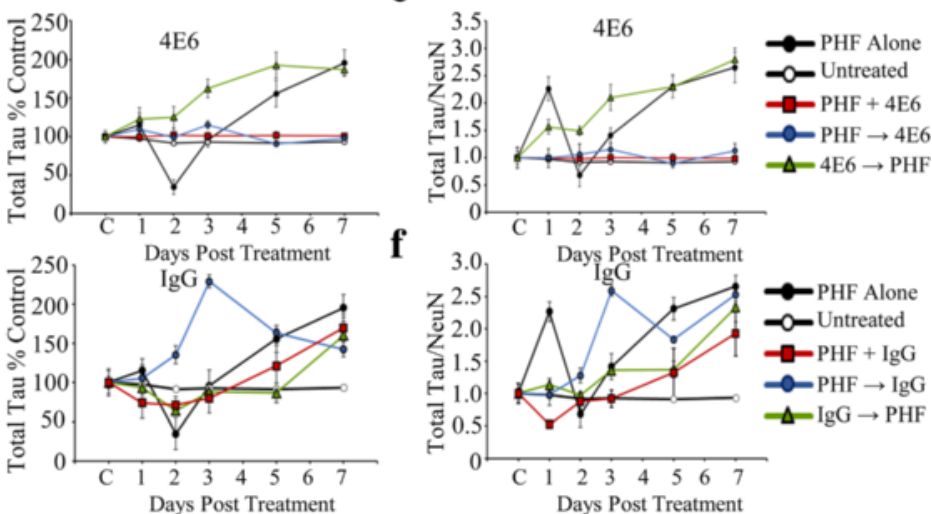

Fig. 8 Low dose PHF ( $1 \mu \mathrm{g} / \mathrm{ml})$ increased the intracellular tau/NeuN ratio, which was prevented by 4E6. a, b Immunoblots for samples exposed to PHF alone or with $\mathbf{a} 4 \mathrm{E} 6$ or $\mathbf{b}$ control IgG1, probed with a pan tau polyclonal antibody. c Quantitation of total tau levels shows that at $1 \mu \mathrm{g} /$ $\mathrm{ml}$, PHF promoted significant increases in intracellular tau (95\% above control, $p<0.0001$ ). For 4E6, the PHF $+\mathrm{Ab}$ and PHF $\rightarrow \mathrm{Ab}$ were significantly lower than the PHF alone samples $(p<0.0001)$. Ab $\rightarrow$ PHF cells were not reduced relative to PHF alone. $\mathbf{d}$ lgG did not prevent against significant increases in total tau after exposure to PHF. e As above, the ratio of tau/NeuN was determined, and again this ratio was significantly increased in PHF alone samples relative to control cells (1.6 fold increase, $p<0.0001)$. Neurons treated with $4 \mathrm{E} 6$ in the PHF + Ab and $\mathrm{PHF} \rightarrow \mathrm{Ab}$, but not $\mathrm{Ab} \rightarrow \mathrm{PHF}$ groups had significantly lower corrected tau levels compared to the PHF alone groups $(p<0.0001)$. $\mathbf{f}$ When adjusted for NeuN levels tau levels in the PHF $+\mathrm{Ab}, \mathrm{PHF} \rightarrow \mathrm{Ab}$, and $\mathrm{Ab} \rightarrow \mathrm{PHF}$ control lgG groups were 0.98, 1.4 and 1.3 fold higher than control, and not significantly different from PHF alone

the PHF alone samples $(p<0.0001$ for both). IgG1 (Fig. 8d) was ineffective under all of the dosing paradigms, as was the $4 \mathrm{E} 6 \mathrm{Ab} \rightarrow$ PHF treatment group.

Tau/NeuN Significant dosing and time effects were found using two-way ANOVA $(p<0.0001$ for both). After normalizing tau levels with NeuN, again the PHF alone cells had significantly increased tau levels relative to control cells (1.6 fold increase, $p<0.0001$ for both, Fig. 8e). Only the 4E6 PHF $+\mathrm{Ab}$ and $\mathrm{PHF} \rightarrow \mathrm{Ab}$ groups had significantly lower normalized tau levels compared to the PHF alone group and did not differ from untreated control samples ( $p<0.0001$ for both). After 7 days in culture, IgG1 samples did not significantly differ from PHF alone (Fig. 8f).

\section{Phospho-Tau}

PHF $10 \mu \mathrm{g}$ In addition to total tau levels, we also assessed the levels of tau phosphorylated at Ser199 (Fig. 9a-c).
Statistical analysis with two-way ANOVA revealed significant effect of dosing method and time $(p<0.0001$ for both). Under the $10 \mu \mathrm{g} / \mathrm{ml}$ PHF conditions, PHF alone and 4E6 $\mathrm{Ab} \rightarrow$ PHF samples had significantly reduced phospho-tau levels relative to untreated cells at day seven (34 and $54 \%$ reduction, $p<0.05$ and 0.001 respectively, Fig. 9d). However, both the $\mathrm{PHF}+\mathrm{Ab}$ and $\mathrm{PHF} \rightarrow \mathrm{Ab}$ treatment groups had significantly higher phospho-tau levels that the PHF alone group $(p<0.001$ and 0.05 respectively, Fig. 9d), and did not differ from untreated cells at day 7. None of the 6B2 (Fig. 9e) or IgG1 (Fig. 9f) dosing groups differed from PHF alone by experimental day 7 .

Phospho-Tau/NeuN Correcting for NeuN levels to take into account PHF toxicity, significant effects of dosing method and time were seen using two-way ANOVA $(p<0.0001$ for both). PHF alone samples showed higher levels of P-Ser199 tau (4.1 fold increase at day $7, p<0.0001)$. Phospho-tau levels in the $\mathrm{PHF}+\mathrm{Ab}$ and $\mathrm{PHF} \rightarrow \mathrm{Ab}$ groups were significantly 
a

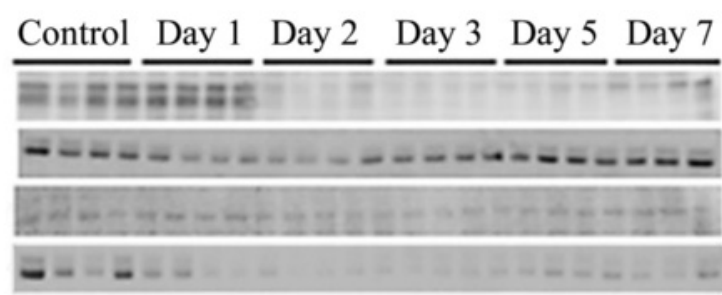

b

Control Day 1 Day 2 Day 3 Day 5 Day 7

$\mathrm{PHF}+6 \mathrm{~B} 2$

$\mathrm{PHF} \rightarrow 6 \mathrm{~B} 2$

$6 \mathrm{~B} 2 \rightarrow \mathrm{PHF}$

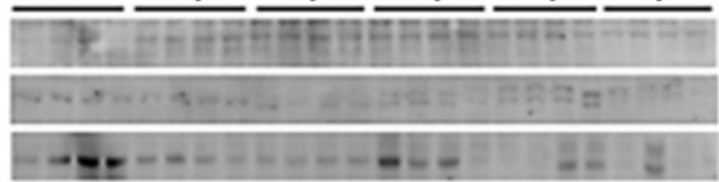

c

Control Day 1 Day 2 Day 3 Day 5 Day 7

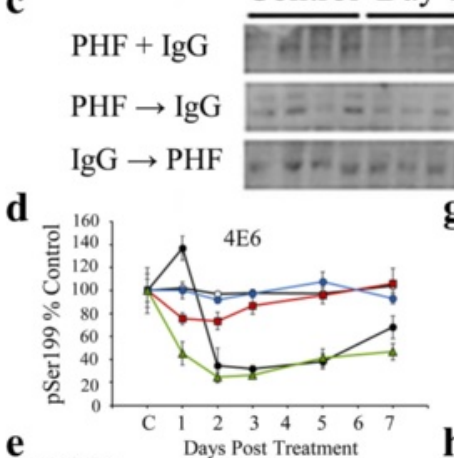

e

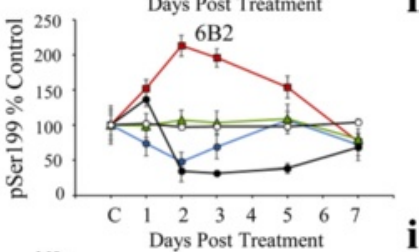

h
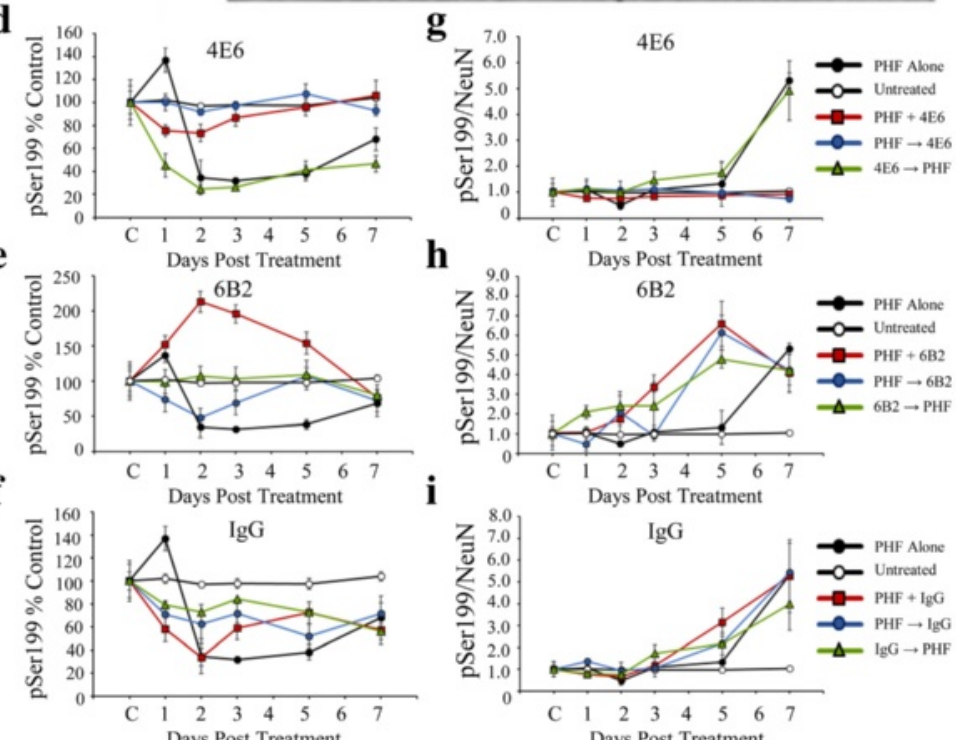

Fig. 9 4E6, but not 6B2, prevented the increase in the phosphorylated tau/NeuN ratio caused by exposure to $10 \mu \mathrm{g} / \mathrm{ml}$ PHF. a-c Samples probed with a polyclonal antibody recognizing tau phosphorylated at Ser199 from cells exposed to PHF alone or PHF in combination with a 4E6, b $6 \mathrm{~B} 2$ or c control IgG1. d PHF alone samples had significantly reduced phospho-tau levels relative to untreated cells (34 \% reduction, $p<0.05)$. For $4 \mathrm{E} 6$, both the $\mathrm{PHF}+\mathrm{Ab}$ and $\mathrm{PHF} \rightarrow \mathrm{Ab}$ treatment groups had significantly higher phospho-tau levels than the PHF alone group ( $p<0.001$ and 0.05 respectively). e In samples treated with a combination of PHF and 6B2, none of the treatment groups were significantly different from PHF alone. $\mathbf{f}$ As with 6B2, uncorrected lgG1 samples showed no significant difference relative to PHF alone. $\mathbf{g}$ Correcting for NeuN levels to take PHF toxicity into account, PHF alone samples had higher ratio of P-Ser199/NeuN, compared to untreated controls (a 4.1 fold increase, $p<0.0001$ ). Phospho- tau levels in the $4 \mathrm{E} 6 \mathrm{PHF}+\mathrm{Ab}$ and PHF $\rightarrow$ Ab groups were significantly lower than the PHF alone samples (91 and $78 \%$ of untreated controls, $p<0.0001$ ). $\mathbf{h}$ When corrected for NeuN levels, $6 \mathrm{~B} 2$ treated cells were not significantly different from PHF alone samples. i When NeuN levels were considered, IgG1 samples were also not significantly different from PHF alone

lower than the PHF alone samples and comparable to untreated controls $(p<0.0001$ for both, Fig. 9g). As with the uncorrected values, none of the 6B2 (Fig. 9h) or IgG1 (Fig. 9i) groups, or the 4E6 $\mathrm{Ab} \rightarrow$ PHF group, were different from PHF alone.
PHF $1 \mu \mathrm{g}$ ANOVA results revealed significant effects of dosing method and time $(p<0.0001$ for both; see Fig. 10a, b for immunoblots). PHF alone samples under the $1 \mu \mathrm{g} / \mathrm{ml}$ dosing conditions had significantly higher phospho-tau levels than untreated control cells $(65 \%$ 

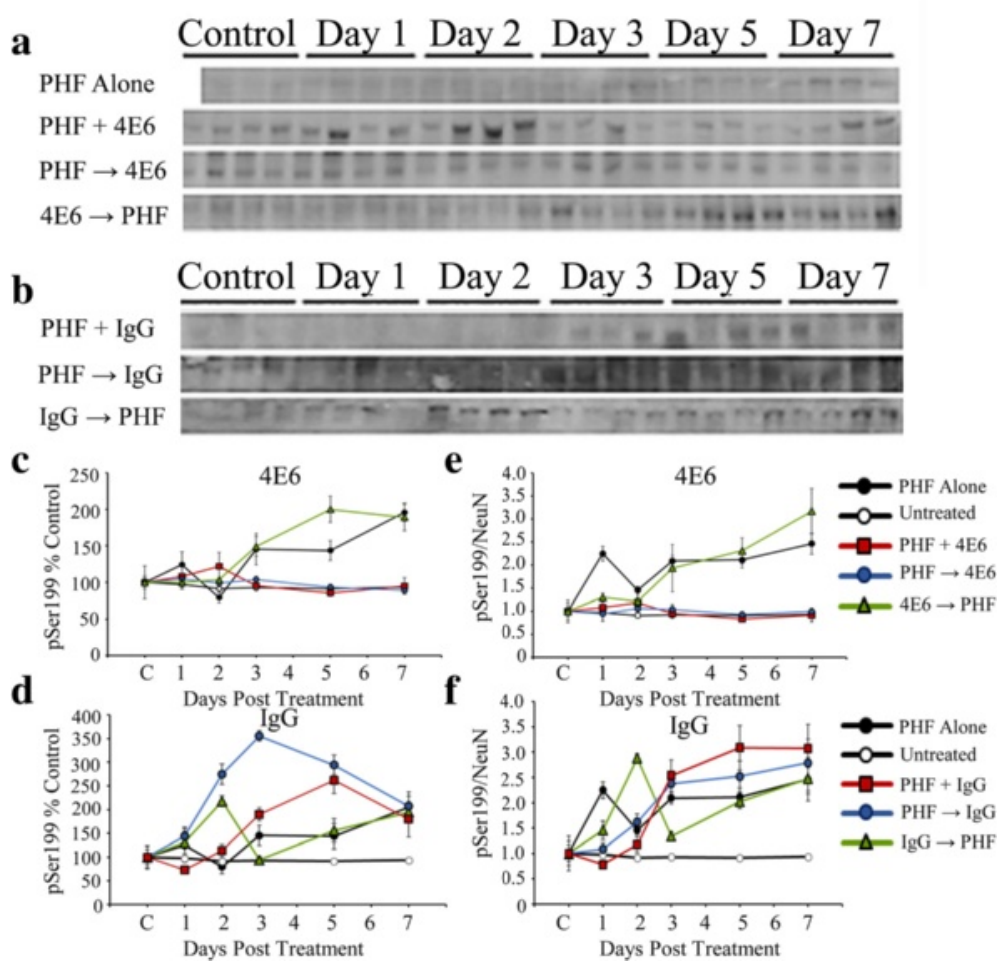

Fig. 10 Low dose PHF $(1 \mu \mathrm{g} / \mathrm{ml})$ increased the phosphorylated tau/NeuN ratio, which was prevented by $4 \mathrm{E} 6$. $\mathbf{a}$, $\mathbf{b}$ Immunoblots of samples treated with $1 \mu \mathrm{g} / \mathrm{ml}$ PHF alone or with a 4E6 or $\mathbf{b}$ lgG1, reacted with a P-Ser199 antibody. $\mathbf{c}$ In cells treated with $1 \mu \mathrm{g} / \mathrm{ml}$ PHF and 4E6, the PHF alone and $\mathrm{Ab} \rightarrow$ PHF groups showed significantly higher phosphorylated tau levels compared to untreated control samples (165 and $185 \%$ above control, $p<0.0001$ for both). The PHF $+\mathrm{Ab}$ and PHF $\rightarrow$ Ab groups were significantly lower (91 and $112 \%$ control, $p<0.0001$ for both) than the PHF alone samples. $\mathbf{d}$ All of the IgG groups showed significantly higher average phospho-tau levels than the untreated controls (79, 119, and $94 \%$ above control, $p<0.0001$ for all) after 7 days. None of the groups were significantly different compared to the PHF alone samples. e As above, NeuN levels were used to control for cell loss, and the pattern of results seen in 4 E6 treated cells remained. The PHF alone and Ab $\rightarrow$ PHF groups had significantly higher phospho-tau/NeuN ratios than untreated samples (1.46 and 1.9 fold higher, $p<0.0001)$. The PHF + Ab and PHF $\rightarrow$ Ab groups had significantly lower phospho-tau levels than the PHF alone samples comparable to untreated controls. $\mathbf{f}$ Correcting for NeuN levels, the same pattern of results in cells treated with control lgG was observed. All groups showed significantly higher levels of phospho-tau/NeuN relative to untreated controls (2, 3.9 and 1.5 fold increase in the PHF $+\mathrm{Ab}, \mathrm{PHF} \rightarrow \mathrm{Ab}$, and $\mathrm{Ab} \rightarrow \mathrm{PHF}$ groups, $p<0.0001$ for all) and no difference relative to the PHF alone samples

above control at day $7, p<0.0001)$. Again, 4E6 was effective in preventing PHF-induced pathological changes under the $\mathrm{PHF}+\mathrm{Ab}$ and $\mathrm{PHF} \rightarrow \mathrm{Ab}$ dosing conditions. Both groups had significantly lower phospho-tau levels than PHF alone at levels comparable to untreated controls $(p<0.0001$ for both, Fig. 10c). However, under the $\mathrm{Ab} \rightarrow$ PHF dosing conditions, 4E6 was ineffective at reducing phospho-tau levels. None of the IgG1 groups (Fig. 10d) differed compared to the PHF alone samples.

Phospho-Tau/NeuN Controlled for NeuN levels, these differences remained. As above, dosing method and time produced significant effects by two-way ANOVA $(p<0.0001$ for both). The phospho-tau levels in the PHF alone samples were 1.5 fold higher than that of the untreated control cells by experimental day seven $(p<0.0001)$. Of the 4E6 dosing paradigms, the PHF + $\mathrm{Ab}$ and $\mathrm{PHF} \rightarrow \mathrm{Ab}$ groups had tau levels comparable to untreated controls and significantly lower than those seen in the PHF alone samples $(p<0.0001$ for both, Fig. 10e). As above, none of the IgG1 groups (Fig. 10f) differed from PHF alone samples.

Together, the total and phospho-tau data show that PHF addition to the cultured neurons increases the levels of these forms of tau in the neurons that survive PHF toxicity. The 4E6 antibody, in addition to preventing PHF-induced toxicity, prevents PHF-induced increases in both total- and phospho-tau, under the $\mathrm{PHF}+\mathrm{Ab}$ and $\mathrm{PHF} \rightarrow \mathrm{Ab}$ conditions. As in the $\mathrm{LDH}$ and NeuN toxicity studies, $4 \mathrm{E} 6 \mathrm{Ab} \rightarrow$ PHF and all three dosing conditions of $6 \mathrm{~B} 2$ and IgG1 were ineffective.

\section{Pattern of PHF and Antibody binding differs depending on dosing method}

To further clarify these efficacy differences, fluorescently labeled PHF and 4E6 or 6B2 were utilized to examine 
whether the treatment paradigm affects the pattern and location of PHF and antibody binding. Primary neurons were incubated using the same dosing methods described above. Confocal images were collected $24 \mathrm{~h}$ after the last treatment was applied. PHF was readily taken up into neurons and could be seen throughout the cells after $24 \mathrm{~h}$ in culture (Fig. 11a-d). In cells from the $\mathrm{PHF} \rightarrow \mathrm{Ab}$ group, we also observed PHF uptake and intracellular distribution. Under this dosing regimen, 4E6 was also internalized and co-localized with the previously added PHF (Fig. 11e-h). However, a different pattern was observed in the $\mathrm{PHF}+\mathrm{Ab}$ group. Under these conditions, PHF and 4E6 were also seen co-

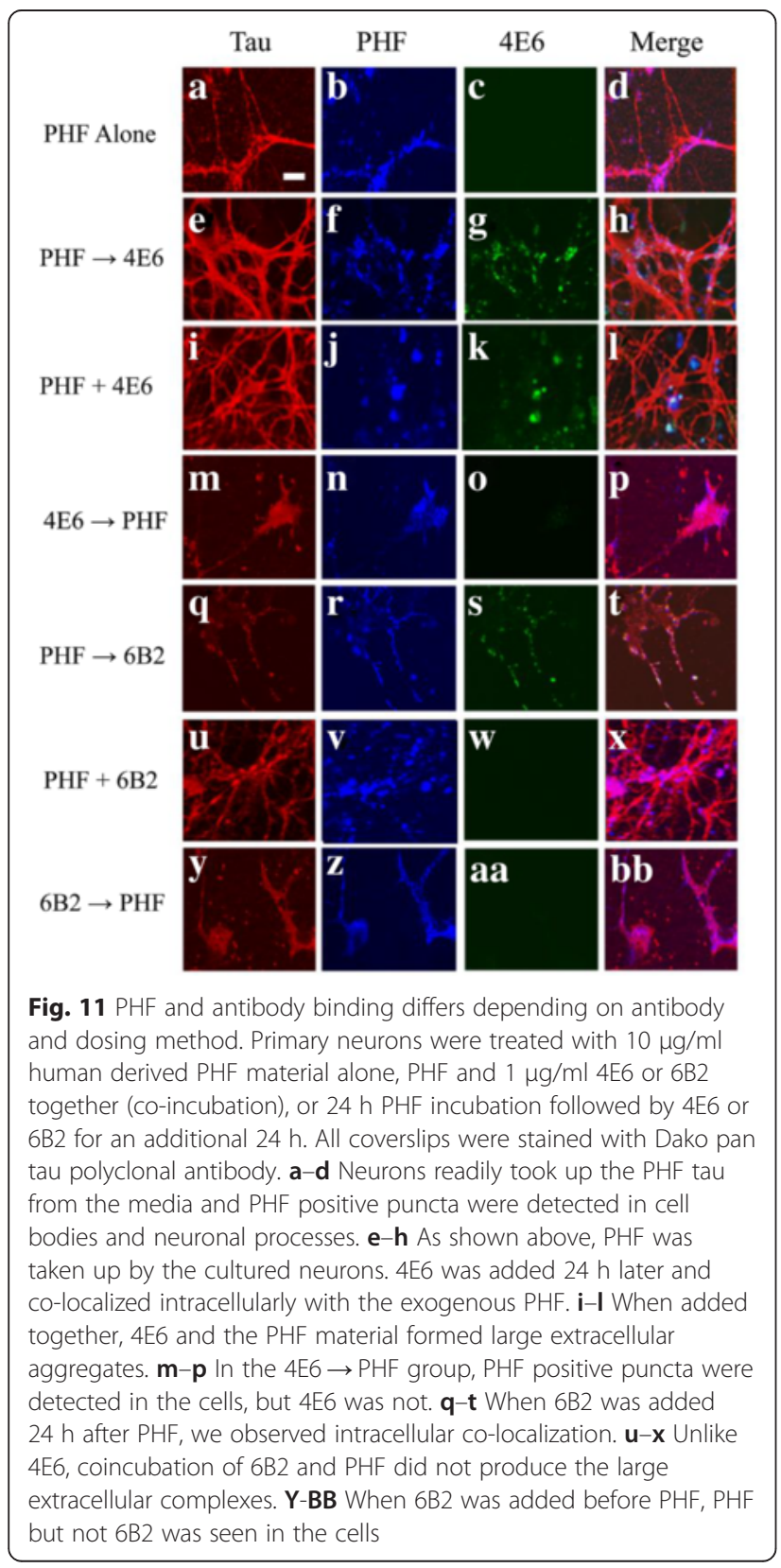

localized, but the PHF-antibody complexes were extracellular (Fig. 11i-l). These results indicate that although these two dosing methods are effective in reducing pathological changes associated with PHF addition, the mechanism of action differs. When cells were incubated with 4E6 prior to PHF, PHF puncta could be seen in the cells, but 4E6 was not apparent, indicating that the antibody may have been cleared from the cell (Fig. $11 \mathrm{~m}-\mathrm{p}$ ). These results help to explain the inefficacy of this paradigm.

We also examined binding between 6B2 and PHF. In the $\mathrm{PHF} \rightarrow \mathrm{Ab}$ condition, intracellular colocalization was observed, however, in the $\mathrm{PHF}+\mathrm{Ab}$ condition the large extracellular PHF-antibody complexes were not seen (Fig. 11q-x). Rather, we observed intracellular PHF material, but 6B2 was not present. In the $6 \mathrm{~B} 2 \rightarrow$ PHF cells, again the PHF material was clearly visible, but the antibody was not (Fig. 11Y-BB).

\section{E6 recognizes primarily solubilized PHF whereas $6 B 2$ binds mainly to aggregated PHF and sarkosyl insoluble tau protein}

To further clarify these intriguing results, binding of the antibodies to tau peptides, solubilized PHF and other tau fractions, was characterized in BIACORE, dot blot and ELISA assays.

\section{BIACORE assay}

Binding to the P-Ser396 peptide was not detected for either antibody. However, for all other epitope peptides, $6 \mathrm{~B} 2$ yielded $K_{D}$ values substantially lower $\left(10^{-9}-10^{-10} \mathrm{M}\right)$ than those seen with 4E6 $\left(10^{-7} \mathrm{M}\right)$, indicating much higher affinity for the immunogen epitope (Table 1).

\section{Dot blot assay}

Binding of 4E6 and 6B2 to PHF was first assayed using a dot blot of different tau fractions (Fig. 4, Fig. 12). The solubilized PHF-, sarkosyl soluble- and sarkosyl insoluble fractions from the same human $A D$ brain were applied to nitrocellulose membrane, which was then incubated with 4E6 or 6B2 (Fig. 12a). 4E6 had higher affinity for solubilized PHF but 6B2 bound better to the sarkosyl insoluble fraction. Neither antibody bound well to the sarkosyl soluble fraction. Both 4E6 and 6B2 showed limited binding to control samples using dot blot (Fig. 12b). No visible reactivity was seen in the sarkosyl soluble fraction, and only minimal reactivity in the other two fractions. Note that the control tissue had very limited if any pathological tau and the pelletable material was much less than in the $\mathrm{AD}$ tissue and likely contains various proteins. Same amount of protein was blotted for AD and control tissue. 
Table 1 Antibody binding to tau peptides

\begin{tabular}{|c|c|c|}
\hline $\mathrm{K}_{\mathrm{D}}$ (BIACORE) & $4 \mathrm{E} 6$ & $6 \mathrm{~B} 2$ \\
\hline \multicolumn{3}{|l|}{30 amino acid peptides } \\
\hline Tau379-408 [P-Ser396/404] & $2.71 \times 10^{-7}$ & $3.95 \times 10^{-10}$ \\
\hline Tau379-408 & $2.12 \times 10^{-7}$ & $2.51 \times 10^{-9}$ \\
\hline \multicolumn{3}{|l|}{23 amino acid peptides } \\
\hline Tau386-408 [P-Ser396/404] & $4.69 \times 10^{-7}$ & $2.39 \times 10^{-9}$ \\
\hline Tau386-408 [P-Ser404] & $2.78 \times 10^{-7}$ & $4.11 \times 10^{-9}$ \\
\hline Tau386-408 [P-Ser396] & ND & ND \\
\hline \multicolumn{3}{|c|}{$\begin{array}{l}\text { The binding of } 4 E 6 \text { and } 6 \mathrm{~B} 2 \text { to tau peptides corresponding to the } 396 / 404 \\
\text { region of the tau protein was examined using a BIACORE assay. Neither } \\
\text { antibody showed binding to the P-Ser396 which differs from previously } \\
\text { published ELISA assays [11]. Using ELISA, 4E6 bound very poorly to the } \\
\text { P-Ser396 peptide coated onto the plate, however, } 6 \text { B2 did show binding. This } \\
\text { may be due to conformational changes which occur in the peptide when } \\
\text { binding to the plate, or differences that occur when the antibody is } \\
\text { immobilized. We observed similar lack of binding of } 4 \text { E6 and } 6 \mathrm{~B} 2 \text { to the } \\
\text { P-Ser396 peptide in solution in competition ELISAs (data not shown), } \\
\text { confirming the accuracy of the BIACORE data. These findings emphasize that a } \\
\text { variety of methods should be used when assessing antibody affinity }\end{array}$} \\
\hline
\end{tabular}

\section{ELISA assays}

Two different ELISA assays were performed to assess binding of 4E6 and 6B2 to different tau fractions from $\mathrm{AD}$ and control brain. In the first, the plate was coated with material from either the sarkosyl soluble, solubilized PHF or sarkosyl insoluble fractions $(1 \mu \mathrm{g} /$ well $)$ and dilutions of antibody were added.

When plates were coated with the solubilized PHF (Fig. 12c), 6B2 showed significantly higher binding to wells coated with material from $\mathrm{AD}$ brain than control for all dilutions up to $1 / 125 \mathrm{k}(p<0.0001-0.05)$ and significantly higher binding that 4E6 to either AD or control at $1 / 200-1 / 5 \mathrm{k}(p<0.0001)$. In contrast, 4E6 did not show significantly higher binding to $\mathrm{AD}$ tau versus control tau at any of the dilutions.

In plates coated with sarkosyl soluble tau (Fig. 12d), low binding was detected even at the highest antibody concentrations. At the $1 / 200$ dilution, $6 \mathrm{~B} 2$ showed significantly higher binding to $\mathrm{AD}$ tau than tau from control brain, and also higher binding than 4E6 to either $\mathrm{AD}$ or control tau ( $p<0.01,0.05$ and 0.05 respectively). None of the other dilutions or conditions showed any significant differences between samples.

Finally, when the plates were coated with the sarkosyl insoluble tau (Fig. 12e), 6B2 showed significantly higher binding than 4E6 to AD tau at the $1 / 200-1 / 125 \mathrm{k}$ dilutions $(p<0.0001-0.05)$ and also significantly higher binding to $\mathrm{AD}$ tau at the same dilutions $(p<0.0001-$ $0.05)$. Again, there was no significant difference in binding to $\mathrm{AD}$ versus control with $4 \mathrm{E} 6$.

A competition ELISA was then performed to determine antibody binding to PHF in solution. In this assay, plates were coated with solubilized PHF as described above, but before antibodies were added aliquots were incubated for $1 \mathrm{~h}$ with increasing concentrations of solubilized PHF $(0.01-1 \mu \mathrm{g} / \mathrm{ml})$. Under these conditions, binding to solubilized PHF markedly inhibited binding of 4E6 to the PHF coated onto the plate, but $6 \mathrm{~B} 2$ binding was not affected. At the highest PHF concentration, 4E6 binding to the wells was reduced by $85 \%$. The IC50 value was determined to be $71 \mathrm{nM}$ (Fig. 12f). In contrast, 6B2 did not show reduced binding to the wells at any PHF concentration. These data indicate that the two antibodies are binding to different tau species within the AD-derived PHF material; 4E6 to solubilized PHF and $6 \mathrm{~B} 2$ to aggregated PHF.

Alone, the ELISA and dot blot binding data are of limited utility for clarifying the efficacy or lack thereof of the antibodies. However, combined with findings from the confocal (Fig. 111 vs. $\mathrm{x}$ ) and biochemical analyses (Fig. 3g) suggests that efficacy of 4E6 and lack thereof for $6 \mathrm{~B} 2$ may be explained by the degree of interaction/ neutralization of PHF. Dot blot assay and ELISA data show that although both 4E6 and 6B2 bind to PHF, the affinity and preferred species differs, with $6 \mathrm{~B} 2$ potentially binding to a more highly aggregated but less toxic tau form (Fig. 12a). In contrast, in all assays 4E6 showed less binding to aggregated tau and preferentially binds to the solubilized PHF. Despite strong binding to the solubilized PHF fraction on dot blots relative to control samples, in ELISA assays 4E6 shows a limited ability to bind to the tau which is aggregated onto the wells. Ab-PHF complexes are not seen with the co-incubation of $6 \mathrm{~B} 2$ and PHF because the latter is in its solubilized form in the culture media which does not bind 6B2 (Fig. 12f). (The solubility of the PHF fraction under experimental concentrations was confirmed using ultra-centrifugation, as described in the Methods section.) $\mathrm{pH}$ changes in endosomes/lysosomes may promote aggregation, resulting in a mixture of soluble and insoluble forms of PHF and, therefore, binding of both antibodies in these vesicles (the $\mathrm{PHF} \rightarrow \mathrm{Ab}$ condition). However, only binding of 4E6 to the solubilized PHF form is beneficial whereas $6 \mathrm{~B} 2$ binding has no effect. This detailed clarification has major implications for the development of tau immunotherapies and for understanding the pathogenesis of tauopathies.

\section{E6 reduces the spread of pathological tau between cell populations}

The ability of 4E6 to prevent the spreading of PHF between cells was assessed using microfluidic axon isolation chambers (Fig. 13a-c shows a schematic of the chambers and neuron growing in the device). Briefly, $\mathrm{Tg}$ cells in one chamber were treated using one of the dosing paradigms described above and the percentage of PHF positive WT cells in the opposite chamber was determined (Fig. 13d-f show PHF positive WT cells). 


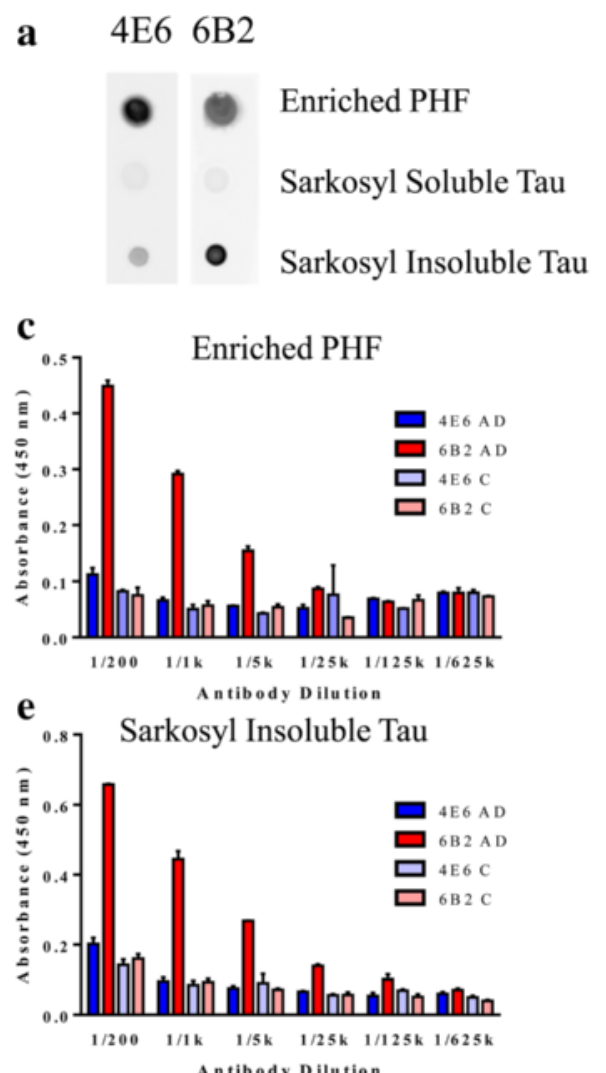

b 4E6 6B2
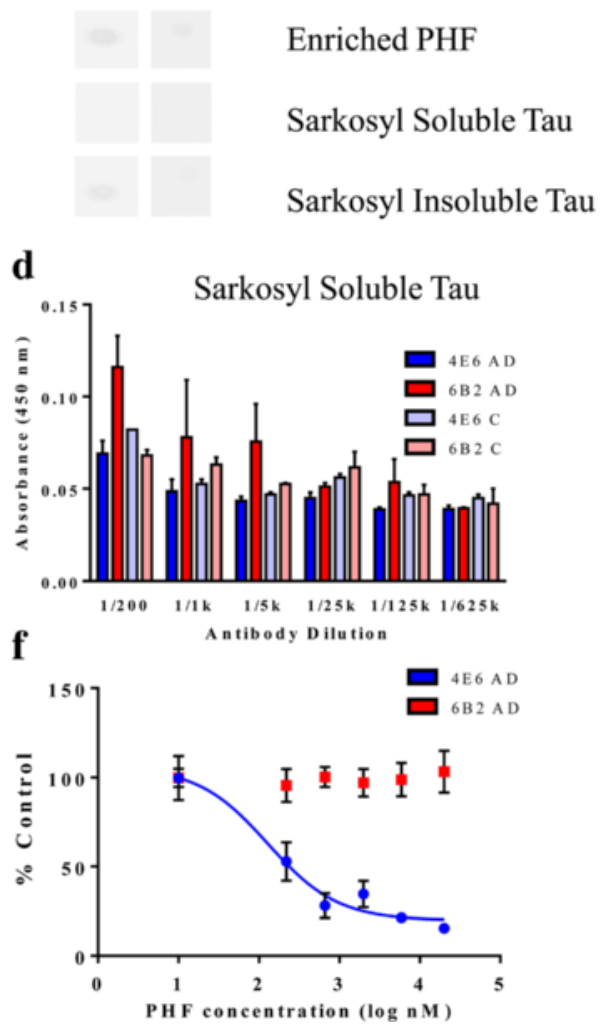

Fig. 12 4E6 and 6B2 differ in their binding to human derived PHF material. a Different tau species were spotted onto nitrocellulose and incubated with either 4E6 or 6B2 as the primary antibody. 4E6 bound better to solubilized PHF but 6B2 reacted more strongly with the sarkosyl insoluble tau fraction. Both antibodies had limited reactivity with sarkosyl soluble tau protein. $\mathbf{b}$ The same three tau fractions were prepared from control brain, and spotted onto nitrocellulose. Neither antibody showed binding to the sarkosyl soluble fraction, and only limited binding to the solubilized PHF and sarkosyl insoluble tau. (Images for all three samples for the 4E6 and 6B2 treated control brains were taken from the same strip, the order has been changed for clarity.) c Plates were coated with solubilized PHF from AD and control brains. 6B2 showed significantly higher binding to AD than control, and than 4E6 to either AD or control at dilutions, 1/200-1/125 ( $p<0.0001-0.05$ ). 4E6 did not show significantly higher binding to AD versus control. d The assay plate was coated with sarkosyl soluble tau from AD and control brains, and serial dilutions of 4E6 and 6B2 were added. At the 1/200 dilution 6B2 showed significantly higher binding to AD than control, and higher binding than 4E6 to either fraction ( $p<0.01,0.05$ and 0.05 respectively). e Assay plates were coated with sarkosyl insoluble tau. $6 \mathrm{~B} 2$ showed significantly higher binding to AD relative to control, and than 4E6 to either AD or control, from dilutions 1/200-1/125 k ( $p<0.0001-0.05$ ). As before, no significant differences between $A D$ and control sample were seen with 4E6. $\mathbf{f}$ Competitive ELISA assays were performed by pre-incubating the antibodies with increasing concentrations of solubilized PHF material (0.01 -1 $\mu \mathrm{g} / \mathrm{ml})$. 6 B2 binding was not inhibited at any PHF concentration. However, 4 E6 binding was inhibited in a dose-dependent manner with an IC50 of $71 \mathrm{nM}$. All of the results show that $4 \mathrm{E} 6$ preferentially binds solubilized tau species, while 6B2 primarily binds to insoluble highly aggregated tau. All columns or points on each graph have SEM error bars, however some of those are too small to be visible

A one-way ANOVA showed a significant treatment effect $(p=0.002$; Fig. 13g). In the PHF alone treated cultures, $24 \pm 3 \%$ of the WT cells contained fluorescently labeled PHF material. When $50 \mathrm{nM}$ of botulinum toxin was added to PHF treated cultures, this was reduced to $4 \pm 0.2 \%(p<0.01)$, indicating that the PHF in the WT cells gets there via synaptic release. PHF $+\mathrm{Ab}$ also significantly reduced the percentage of PHF positive WT cells to $15 \pm 2 \%(p<0.05)$. PHF $\rightarrow$ Ab treatment groups showed a lower percentage of PHF positive WT cells, $17 \pm 4 \%$ although the results did not reach significance. However, as expected based on other results, there was no significant change in the percentage of PHF positive WT cells under $\mathrm{Ab} \rightarrow$ PHF conditions, further confirming the inefficacy of this approach.

\section{E6 is effective intra- and extracellularly in blocking PHF- induced toxicity and associated tau pathologies}

To further investigate whether timing of antibody and PHF affect the mechanism of action, an additional group of cells was plated and dosed as described above. However, when 4E6 was applied to the cultures, $1 \mu \mathrm{g} / \mathrm{ml}$ of dansyl cadaverine (DC) was added as well (Fig. 14). DC is an inhibitor of clathrin (receptor)-mediated endocytosis 
$\mathbf{a}$
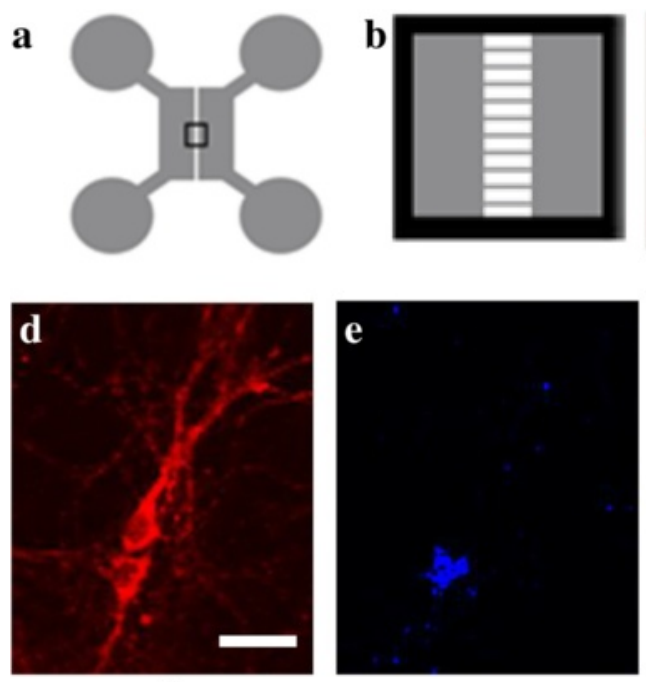

g
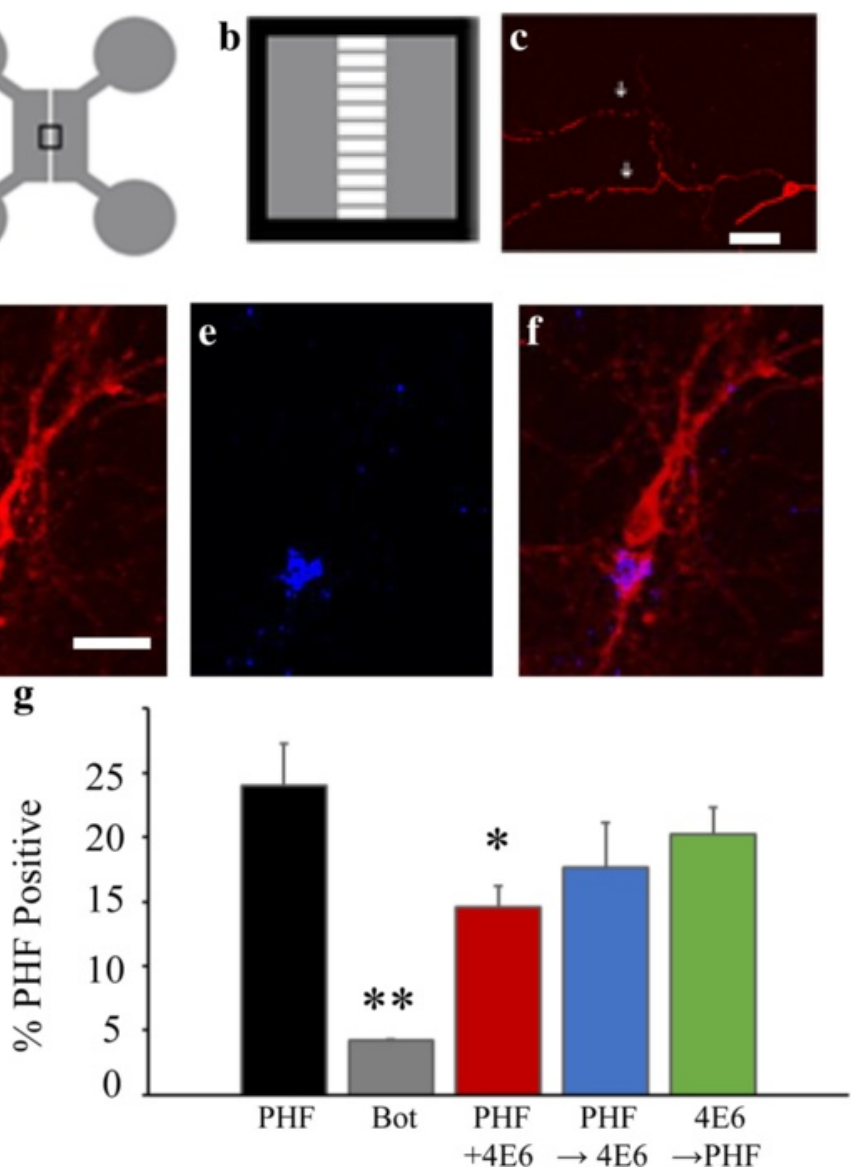

Fig. 134 E6 reduced the spread of tau between neurons. a, b Schematic of microfluidic chambers, showing the reservoirs that the cells were added to. Panel $B$ is a magnification of the box in panel $A$ showing the microgrooves which connect the two reservoirs. c Confocal image showing axons growing through the microgrooves. Cell is stained with pan-tau antibody. (scale bar $=150 \mu \mathrm{m}) \mathbf{d}$-f f Fluorescently labeled PHF material $(1 \mathrm{\mu g} / \mathrm{ml})$ was added to the chamber containing JNPL3 cells. Coverslips were fixed and stained with an antibody recognizing total tau. Stained wild-type neurons from the opposite chamber are visualized in $\mathbf{d}$, and one of them has prominent PHF puncta in the cell body as seen in E. Merged image of $\mathbf{d}$ and $\mathbf{e}$ is depicted in $\mathrm{F}$ (scale bar $=50$ ). $\mathbf{g}$ Neurons in the first chamber were treated with PHF and $4 \mathrm{E} 6$ in combination as described in Fig. 4b. Following addition of the antibody, cells were incubated for a further $72 \mathrm{~h}$. Number of cells in the opposite side containing PHF puncta was recorded. Botulinum toxin was used as a negative control. In the PHF alone condition, $24 \%$ of cells were PHF positive. Incubation with botulinum toxin reduced this percentage to $4 \%(p<0.01)$. In the $\mathrm{PHF}+\mathrm{Ab}$ group this was reduced to $14.6 \%(p<0.05)$, and $17.6 \%$ in the PHF $\rightarrow$ Ab condition. *: $p<0.05, * *: p<0.01$

and in previous experiments, not using exogenous PHF, has been shown to block uptake of 4E6 [10]. Thus, these experiments allowed us to confirm whether antibody internalization is required to prevent PHF-induced pathology. When total tau levels were examined by immunoblot, there was no significant difference between samples incubated with or without DC under either dosing conditions (data not shown). In PHF $+\mathrm{Ab}$ samples, addition of DC did not change NeuN levels (data not shown) or tau/NeuN ratio (Fig. 14a). However, when cells in the $\mathrm{PHF} \rightarrow \mathrm{Ab}$ condition were incubated with $\mathrm{DC}$, a significant decrease in NeuN was observed when compared to cells without DC (NeuN reduced to $32 \%$ of control, $p=0.00005)$. Further, the ratio of $\mathrm{Tau} / \mathrm{NeuN}$ was significantly shifted in the PHF $\rightarrow$ Ab samples where DC was added (1.18 for cells without DC and 2.95 for DC treated cells, $p=0.008$; Fig. 14b). These results indicate that under conditions where 4E6 and PHF are added together, receptor-mediated internalization (via Fc receptors based on our prior findings [10]) is unnecessary for the antibody to prevent PHF induced pathological changes. In contrast, once the PHF material has been taken up by the neurons, blocking antibody internalization reduces its efficacy in preventing PHF toxicity.

\section{Discussion}

Our findings indicate that two antibodies against the same epitope region have very different effects on cognition in a mouse model of early stage tauopathy. Both antibodies have phospho-selectivity for the immunogen 

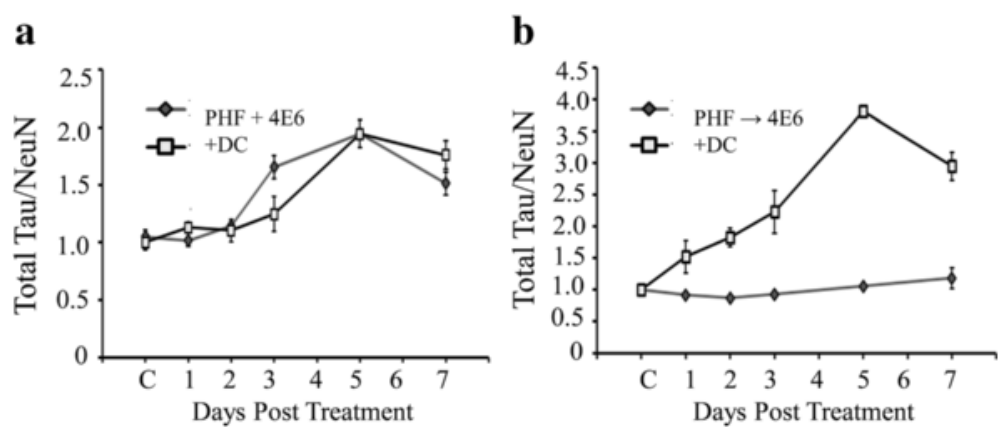

Fig. 14 Mechanism of action is influenced by dosing method. PHF $(10 \mu \mathrm{g} / \mathrm{ml})$ was added to JNPL3 neurons, with 4E6 added either at the same time or $24 \mathrm{~h}$ later. In additional cultures, $1 \mathrm{\mu g} / \mathrm{ml}$ dansyl cadaverine (DC), an inhibitor of clathrin mediated endocytosis, was also added along with 4E6 to determine whether antibody internalization is necessary for 4E6 to exert its effects. $\mathbf{a}$ In the PHF + Ab treatment group, addition of DC did not affect the NeuN corrected tau levels, indicating that the antibody is working extracellularly. $\mathbf{b}$ When DC was present in the PHF $\rightarrow$ Ab condition, total tau/NeuN ratios was shifted beginning at $24 \mathrm{~h}$, and the relative tau levels were significantly higher after 7 days. Hence, under these conditions, the antibody is working intracellularly

but differ in many ways. Interestingly, the lower affinity antibody, 4E6, is effective in acutely improving spatial learning and memory and reducing soluble phospho-tau, whereas the higher affinity antibody, 6B2, is ineffective. Importantly, we further show identical efficacy differences in a primary neuronal tauopathy culture model treated with paired helical filaments (PHF) isolated from an Alzheimer brain. This indicates that the ex vivo culture model has similar predictive validity as the mouse model although the measured parameters are not comparable.

Modest tau pathology was detected by immunohistochemistry in brains of htau mice, and the treatment and control groups did not appear to differ. Under such conditions of early stage tau pathology, it is easier to quantitate early tau pathology on western blots than by immunohistochemistry, and on such blots insoluble tau protein was clearly present in the 12-13 month old htau mice. Neither tau antibody induced changes in insoluble tau levels as measured by human specific tau antibody (CP27), although 4E6 markedly improved spatial learning and memory. Analyses of the soluble tau fraction revealed that these cognitive benefits were associated with reduced levels of phospho-tau protein (PHF-1 reactive). It is likely that under such acute treatment conditions, global changes in insoluble tau levels may not be readily achievable, whereas soluble pathological tau protein should be more amenable to clearance. Indeed, the PHF1 antibody recognizes a phospho-tau epitope within the same region as 4E6, which may explain why this tau fraction is preferentially cleared. However, it does not appear to be oligomer specific clearance, as we did not observe any differences in T22 immunoblots between 4E6 and IgG-treated mice.

We did not observe a functional rescue of associative fear memory following acute treatment with either 4E6 or 6B2. There are many possible explanations for this. First, the training protocol used may have been too 'strong' to detect a subtle memory deficit. This may be particularly important because the overall tau pathology that we observed, although present, was mild. In our prior studies we only detected associative memory deficits in aged mice with greater levels of tau pathology [24].

Despite the different model systems used, the findings obtained from ex vivo and in vivo experiments are consistent and not model dependent, which supports their validity. In both cases, 4E6 shows efficacy in preventing tau pathology and associated toxicity/cognitive impairment, while $6 \mathrm{~B} 2$ does not.

An insight into the relevant tau species was obtained from ELISA and dot blot studies of antibody binding to soluble, solubilized, or aggregated human tau species. 4E6 recognizes primarily solubilized PHF, in an ELISA and dot blot assay, which may explain lack of more global tau changes in the animals under such acute conditions. Mice at this age with modest tau pathology may be ideal to assess acute effects of therapies, particularly under pairwise cognitive comparison as used herein, which improves the sensitivity of detecting beneficial effects. Such in vivo learning and memory benefits by $4 \mathrm{E} 6$ and lack thereof for $6 \mathrm{~B} 2$ in the htau mice are in agreement with the efficacy results in vivo and in the tauopathy culture model. Interestingly, soluble tau species have recently been linked to LTP and memory in htau mice [25].

Although the ELISA and dot blot assays provided useful information on the tau binding properties of 4E6 and $6 \mathrm{~B} 2$, the data obtained from confocal imaging was of greater value in determining mechanism and possible explanations for the differences in efficacy. With coincubation in the culture assay, extracellular complexes 
of exogenous PHF and 4E6 formed (Fig. 11l) as 4E6 binds to soluble PHF. This complex formation neutralized PHF and prevented its uptake. However, with 6B2, such complexes did not form, as 6B2 does not bind well to solubilized PHF, and PHF was detected intraneuronally (Fig. 11x). This indicates that 6B2 could not prevent PHF uptake and toxicity. These results support that antibodies can be beneficial while working in the interstitial space between cells. In the living brain, these tauantibody complexes could then be taken up and cleared by microglia as we have seen previously [11] and others have studied more extensively [26, 27].

Alone, addition of 10 or $1 \mu \mathrm{g} / \mathrm{ml}$ PHF dosedependently induced cell loss, as measured using LDH and NeuN levels, as well as increased total and phosphorylated tau in the remaining neurons. It spread between cell populations, through release and subsequent uptake by other neurons. To test the efficacy of our antibodies, we utilized three different dosing methods differing in the timing of tau and antibody administration. For one of the antibodies, 4E6, two of these methods, addition of the PHF and antibody together, and addition of 4E6 24 h after PHF, prevented PHF toxicity, seeding, and spread. Interestingly, although similarly effective, the mechanism through which the protection occurs differed between the dosing paradigms.

When 4E6 or 6B2 were added $24 \mathrm{~h}$ after PHF, these colocalized intracellularly with PHF but only 4E6 prevented PHF toxicity. Based on the confocal data from the co-incubation experiments, as well as dot blot and ELISA data, 4E6 binds better to solubilized PHF than 6B2 which reacts better with aggregated $\mathrm{PHF}$ and insoluble tau. Hence, this feature may explain the intracellular efficacy of 4E6. It may prevent PHF polymerization, facilitating access of lysosomal enzymes to clear PHF and/or directly neutralizing soluble PHF and preventing toxicity. However, 6B2-PHF binding may be inert without promoting disassembly. Furthermore, due to poor binding it may be unable to prevent PHF fibril formation and/or toxicity of soluble PHF.

Together, these findings explain the therapeutic efficacy of 4E6. It is capable of both extracellular blockage and intracellular clearance of PHF. Our previous data indicates that 4E6 enters the endosomal/lysosomal system within tauopathy neurons and promotes clearance of native tau, possibly by preventing aggregation $[10,11,13]$. Other groups have observed internalization of tau antibodies [9, 12], and lysosomal colocalization [9]. Further, neuronal colocalization between antibody, target, and endosomal/lysosomal markers has been seen for $\alpha$-synuclein antibodies in a PD mouse model [28]. In other experiments, tau antibodies are able to block the uptake of pathological tau or improve experimental outcomes without apparently entering neurons [15-17].
Whether antibodies are taken into neurons is likely influenced by several factors including, charge, target and Fc receptor affinity, and as data presented herein suggests, location of the target and timing of antibody addition.

In contrast, pre-incubation with 4E6 was ineffective at reducing toxicity or seeding of tau pathology. A likely reason is the relative lack of the target epitope under these conditions. Previously, we showed that neuronal 4E6 uptake correlates highly with pathological intracellular tau levels [10]. When the antibody is added first, efficacy requires retention in neurons until PHF addition $24 \mathrm{~h}$ later. However, a shortage of the target means the antibody will remain unbound, and more prone to degradation or recycling out of the cell, as seen via confocal imaging. Although 4E6 was ineffective under these conditions, it does not rule out prophylactic administration of tau antibodies, as circulating antibodies could prevent disease initiation by clearing early-stage tau aggregates. Exogenous antibodies have a half-life of one to three weeks and lower doses could be used in presymptomatic individuals at risk.

Notably, the different efficacies between dosing methods were also observed in the spreading assay using the microfluidic chambers. In both model systems, only the co-incubation dosing or $\mathrm{PHF} \rightarrow \mathrm{Ab}$ was effective, whereas $\mathrm{Ab} \rightarrow \mathrm{PHF}$ was not.

In our previously published findings [11], both 4E6 and 6B2 showed efficacy in preventing increased phospho-tau levels in a brain slice model, in contrast to 6B2's ineffectiveness in the primary neurons in the current study. There are likely multiple factors which contribute to these differences. In the slice culture system, treatment with antibodies lasted for up to 6 weeks and no exogenous tau was introduced in that system. In the present experiments, a much shorter time scale is used (7 days as opposed to six weeks) and we are utilizing PHF tau isolated from a human AD brain. The tau found in the PHF fraction also represents a different stage of tangle formation. Furthermore, the PHF isolated from the AD brain may have additional posttranslational modifications not present in the endogenous tau of the slices. Differences in cell health over the course of the experiments are likely also influenced by the culture model. In the primary cultures, neurons lack the trophic support provided by glial cells, which are present in the brain slices.

We have shown that antibody uptake into neurons can be blocked with an antibody against FcII/III receptors or with dansyl cadaverine, which blocks receptor-mediated endocytosis [10]. Under the co-incubation conditions, blocking antibody uptake had no effect on the outcome. However, when 4E6 was added 24 h after PHF addition, blocking its uptake prevented its beneficial effects. These 
findings confirm that under co-incubation conditions, the antibody is working extracellularly but when it is added $24 \mathrm{~h}$ after PHF, its effects are intracellular.

We have previously shown that both antibodies are taken up into tauopathy neurons in brain slice- and primary cultures, in which they colocalize with tau aggregates in the endosomal-lysosomal system $[10,11]$. Furthermore, $6 \mathrm{~B} 2$ and its single chain variable fragment derivative can be used to image tau lesions in vivo and end up in the same neuronal compartments after peripheral injection [14]. Such uptake and colocalization is by itself not an indication of efficacy but we have shown that prevention of 4E6 neuronal uptake blocks acute antibody-mediated tau clearance [10]. The culture data confirms such intracellular clearance and additionally shows prevention of neurotoxicity by 4E6 in a different culture model, which is more relevant to human disease as AD derived PHF material is used. Furthermore, in our PHF-treated primary culture study, 6B2 was ineffective under various experimental conditions using multiple outcome measures. Overall, 4E6 may be better suited as a therapeutic antibody targeting soluble tau species and $6 \mathrm{~B} 2$, or ideally its smaller derivatives with better access to the target, useful as an imaging probe for insoluble tau lesions.

Specifically, the ex vivo culture model shows that 4E6, a monoclonal tau antibody targeting the phospho-serine 396/404 region prevented toxicity and reduced tau levels induced by the addition of Alzheimer's brain-derived PHF material. Importantly, another tau monoclonal, $6 \mathrm{~B} 2$, which has substantially higher affinity for the tau peptide immunogen and aggregated PHF tau than 4E6, was ineffective under these experimental conditions. Further analyses revealed that 4E6 had higher affinity than 6B2 for the solubilized PHF that was used to promote toxicity in cultured neurons. This likely explains the efficacy of the former antibody and lack thereof for the latter. These findings have major implications for the development of passive tau immunotherapies. Efficacy cannot be predicted by affinity to the immunogen alone or to aggregated tau, but has to be determined in biological models of tau pathology. Combined with imaging data, these results provide information on how affinity and efficacy relate.

Other tau immunotherapy studies have reported efficacy differences between antibodies recognizing epitopes of different sequences of tau and one study between different isotypes of two antibodies of similar affinity against the same epitope (for review see [5]). $4 \mathrm{E} 6$ and $6 \mathrm{~B} 2$ are of the same isotype, IgG1, and our findings show for the first time that subtle difference in epitope recognition can profoundly affect efficacy. Importantly, we have confirmed and provided mechanistic insight into these in vivo differences in a disease relevant ex vivo neuronal culture model, in which we promote tau pathology with Alzheimer's brain-derived PHF in primary neurons expressing familial tau mutation. Hence, the contrasting efficacies are seen consistently in different models with or without tau mutation and may have major therapeutic implications for both familial and sporadic tauopathies. The models employed have strong construct and face validity as they are based on sound theoretical rational as normal or familial (mutated) human tau is being expressed, and have the key features associated with tauopathies, namely tau aggregation, toxicity, and associated cognitive impairments in the animals. The human PHF culture model has strong predictive validity for the outcome in the animal model, but it remains to be seen if this holds up in clinical trials.

\section{Conclusions}

Overall, these findings indicate that antibody efficacy cannot be predicted by affinity to the immunogen alone or broad reactivity with various typical pathological forms of tau but has to be determined in biological models of tau pathology. Binding to solubilized PHF rather than aggregated PHF or insoluble tau predicts efficacy in these ex vivo PHF seeding and spreading culture models and in a tauopathy mouse model. 4E6 promotes clearance of pathological tau and thereby prevents its toxicity via intra- and extracellular pathways. Such wide spectrum antibodies are likely to be more efficacious than antibodies that can only work outside neurons. Importantly, the ex vivo findings fit nicely with acute antibody efficacy in vivo in improving cognition, which was associated with clearance of soluble phospho-tau.

Future studies should consider that relatively subtle epitope differences, even within the same tau region, can have major implications for therapeutic outcome, and that higher affinity for the immunogen or various pathological forms of tau does not necessarily translate into better efficacy. Considering that antibody humanization and scale up for clinical trials may alter binding characteristics compared to the original mouse monoclonal antibody, these findings may have major implications for ongoing and future clinical trials of tau antibodies.

\section{Methods}

\section{Animals and in vivo experimental design Ex vivo}

Pups from the homozygous JNPL3 mouse line (human 0N4R with P301L mutation, Taconic) were collected at postnatal day zero for primary cultures [29]. This model was chosen because of its robust tau expression and phosphorylation even at an early age.

For tau spread experiments, wild type (WT) pups from the same strain background were also utilized. 
All breeding animals were monitored closely during the progression of pregnancy to ensure that pups were collected on the day of birth to make certain that each culture was at the same developmental age when plated.

\section{In vivo}

The htau model (Jackson Laboratories, stock\#004808; [20]), expresses all six isoforms of unmutated human-tau protein on a null mouse-tau background and develops progressive tau pathology. This model was utilized in the whole animal treatment studies due to its more naturalistic tau expression relative to other tauopathy models. It was used to assess therapeutic benefits of acute passive immunotherapy with tau monoclonal antibodies 4E6G7 (4E6) or 6B2G12 (6B2), purified from our hybridoma by Genscript (Paramus, NJ), relative to IgG-injected (Equitech Bio Inc.) controls. In the 4E6 study, 18 mice (7 males $(\mathrm{M})$ and 11 females $(\mathrm{F}))$ received 4E6 and 18 mice (8 $\mathrm{M}$ and $10 \mathrm{~F}$ ) control IgG. No animals died during the study but, four mice in each group were excluded from all analysis, due to no human-tau expression, despite containing the transgene, leaving 14 4E6 mice (4 M and $10 \mathrm{~F}$ ) and 14 IgG mice (7 M and $7 \mathrm{~F}$ ) for analysis.

In the 6B2 study, only female mice were enrolled, 12 per group. As in the 4E6 study, no animals died during the experiment, but four IgG-treated and one 6B2treated mouse were excluded due to lack of human-tau expression, leaving $116 \mathrm{~B} 2$ treated mice and 8 IgG controls for analysis.

At the start of the study, the htau mice were 11-12 months of age and split into two groups with similar cognitive and motor status before receiving three antibody injections and going through retesting on the same behavioral tests and an additional fear-conditioning test, followed by brain extraction for tissue analysis. The mice went through adaptation and pre-tests using Rotarod, Open Field and Closed Field Symmetrical Tests on days 1-10 as we have detailed previously [30], followed by antibody injection on days 11 and 14 and retesting on days 15-18. Third injection was delivered on day 24 followed by fear-conditioning test on days $27-28$, and perfusion on day 30.

All animals were housed at NYU School of Medicine animal facilities and cared for by the veterinary staff in AAALAC-approved facilities. All the procedures were approved by the Institutional Animal Care and Use Committee (IACUC) committee of the university, and are in accordance with NIH Guidelines, which meet or exceed the ARRIVE guidelines.

\section{Isolation of paired helical filaments (PHF)}

PHF tau was extracted from the brain of a human AD patient as described by others [23] but with a few modifications (See Fig. 4) to create the three fractions used to treat cells and assess antibody binding via dot blot. Briefly, the tissue was homogenized in buffer (pH 6.5; $0.75 \mathrm{M} \mathrm{NaCl}, 1 \mathrm{mM}$ EGTA, $0.5 \mathrm{mM} \mathrm{MgSO}$, and $100 \mathrm{mM}$ 2-( $N$-morpholino) ethanesulfonic acid) and centrifuged at $11,000 \times \mathrm{g}$ for $20 \mathrm{~min}$ to remove debris. The supernatant was centrifuged for an additional $60 \mathrm{~min}$ at $100,000 \mathrm{x} \mathrm{g}$. The resulting pellet was resuspended in extraction buffer (10 mM Tris; $10 \%$ sucrose; $0.85 \mathrm{M} \mathrm{NaCl}$; and $1 \mathrm{mM}$ EGTA, $\mathrm{pH}$ 7.4) and centrifuged at $15,000 \mathrm{x}$ g for $20 \mathrm{~min}$. The supernatant was retained and incubated with $1 \%$ sarkosyl at room temperature. This supernatant was then centrifuged for $60 \mathrm{~min}$ at $100,000 \mathrm{x}$ g. The supernatant from this step was retained and designated the sarkosyl soluble fraction. The pellet was resuspended in $50 \mathrm{mM}$ Tris- $\mathrm{HCl}$ buffer and designated the sarkosyl insoluble fraction. For the cell culture experiments this fraction was first heated briefly to $37^{\circ}$ $\mathrm{C}$ and then dialyzed in PBS, yielding the enriched PHF fraction. The heating and dialysis promote solubility and result in solubilized enriched PHF tau fraction which was used in the experiments. Under our conditions, the PHF fraction is soluble at the doses used (1 and $10 \mu \mathrm{g} /$ $\mathrm{ml})$. Prior work by others indicates that PHF can be soluble at least up to $100 \mu \mathrm{g} / \mathrm{ml}$ [23]. In addition, other groups have demonstrated that dilution of tau filaments leads to their disassembly [31-33].

In order to assess the solubility of the PHF material at the concentrations used, we prepared a $10 \mu \mathrm{g} / \mathrm{ml}$ solution and subjected it to centrifugation at $100,000 \mathrm{x} g$ for $60 \mathrm{~min}$. Following this spin, the supernatant was removed. Under these conditions, no visible pellet was seen.

Brain tissue from a control brain was processed in the same way. Note that the control tissue has very limited if any pathological tau and the pelletable material is much less than in the AD tissue and likely contains various proteins. Same amount of protein was used in each assay for $\mathrm{AD}$ and control tissue.

\section{ELISA assays}

ELISA assays were performed as described [11]. Briefly, 96 well plates were coated with one of three different tau fractions (sarkosyl soluble, solubilized PHF, or sarkosyl insoluble) derived from either AD or control brains dissolved in $50 \mathrm{mM}$ carbonate buffer ( $\mathrm{pH}$ 9.6). Plates were blocked using Superblock (Fisher Scientific) for one hour at room temperature. After blocking, serial dilutions (1/200-1/625,000) of the antibodies were added to the plate and incubated for $2 \mathrm{~h}$ at room temperature. All antibodies were adjusted to a concentration of $1 \mathrm{mg} / \mathrm{ml}$ before dilution. Plates were washed with $0.1 \%$ TBS-T and HRP-conjugated mouse secondary antibody (1:5000) was added for one hour. 
TMB peroxidase EIA reagent (Fisher Scientific) was used to develop the signal and stopped with $2 \mathrm{~N}$ sulfuric acid. Absorbance at $450 \mathrm{~nm}$ was read using a BioTek Synergy 2 plate reader.

For the competitive ELISA assays, a single antibody concentration was utilized (1:1000 dilution of $1 \mu \mathrm{g} / \mathrm{ml}$ stock). Aliquots of 4E6, 6B2 and IgG1k isotype control (eBioscience) were incubated for $30 \mathrm{~min}$ with increasing concentrations of the human derived solubilized PHF material prior to plating.

\section{Fluorescent labeling}

4E6 and human derived solubilized PHF material were labeled using Alexa Fluor 488 and 647 labeling kits, respectively as per kit instructions.

\section{Primary neuronal cultures}

Cultures were prepared as described from the cortex and hippocampus of p0 pups, with all components purchased from Invitrogen unless otherwise detailed [10]. After $24 \mathrm{~h}$ in culture, plating media was removed and neuronal media (Neurobasal A, $1 \mathrm{ml} \mathrm{B27,} 17 \mu \mathrm{l}$ basal medium Eagle) added. As in previous studies, the purity of the neuronal cultures was assessed using confocal imaging. Under these conditions, about $95 \%$ of cells are neurons (positive for NeuN) [34].

For cultures grown in microfluidic chambers the same procedure was utilized. Cells from the JNPL3 mice were plated on one side of the axon isolation device, and were allowed to incubate for $72 \mathrm{~h}$. Following this period, brains from WT animals were harvested and those cells plated on the opposite side.

\section{Ex vivo experimental design}

Primary neuronal cultures were prepared as described and allowed to recover in culture for one week prior to treatment [10]. Cells were incubated with either 1 or $10 \mu \mathrm{g} / \mathrm{ml}$ of the human derived PHF material, with cells and culture media collected at 1, 2, 3, 5 and 7 days. For cultures being treated with a combination of PHF and antibody (4E6, 6B2, or IgG), three different treatment strategies were used. In the first, PHF material was added and allowed to incubate with the cultures for 24. h. Following this period, the cells were washed with neuronal media, and fresh media containing $1 \mu \mathrm{g} / \mathrm{ml}$ of antibody was added ( $\mathrm{PHF} \rightarrow \mathrm{Ab}$ ). In the second, PHF material and antibody were added to the culture media simultaneously ( $\mathrm{PHF}+\mathrm{Ab})$. The third dosing strategy is the inverse of the first, antibody was added $24 \mathrm{~h}$ prior to PHF (Ab $\rightarrow$ PHF). See Fig. 4 for a summary of dosing methods. In experiments using dansyl cadaverine (DC; Fisher Scientific), the same methods were employed with $1 \mu \mathrm{g} / \mathrm{ml} \mathrm{DC}$ added along with the antibody.

\section{LDH assays}

Media was collected from all treatment groups after seven days in culture. LDH levels in the media were determined using a Roche cytotoxicity detection kit. Values obtained from treated samples were compared to media collected on day zero to assess toxicity. Media from a set of untreated cells was also collected to examine the normal changes in cell health over the culture period. Treated samples, controls and blanks were added to a 96 well plate and the detection reagents were added as per the instructions. Plates were incubated for $20 \mathrm{~min}$ at $37{ }^{\circ} \mathrm{C}$ and read using a BioTek Synergy 2 plate reader.

\section{Microfluidic chambers and tau spreading}

Following the addition of the WT cells, cultures were given one week in culture to stabilize. The same three treatment methods were utilized. PHF and 4E6 $(1 \mu \mathrm{g} /$ $\mathrm{ml}$ each) were added to the chamber containing JNPL3 cells, while the opposing chamber containing WT cells was left untreated. As a negative control, one group of cells was incubated with $1 \mu \mathrm{g} / \mathrm{ml}$ of the PHF material and $50 \mathrm{nM}$ of botulinum toxin (generously provided by Dr. Edwin Vasquez-Cintron, NYU School of Medicine). The toxin was chosen due to its ability to prevent membrane fusion and the release of membrane bound vesicles, and thus prevent release of PHF tau into the opposite chamber. In neuronal cultures, botulinum toxin has also been shown to prevent the spread of mutant huntingtin by blocking synaptic vesicle release [35]. In all groups, after the final treatment, cells were maintained in culture for a further $72 \mathrm{~h}$. Coverslips were then fixed and stained for antibodies recognizing total tau. The percentage of cells in the contralateral chamber containing labeled PHF material was determined for each treatment group.

\section{Immunohistochemistry \\ Animals}

Following behavioral testing, mice were anesthetized with ketamine/xylazine $(250 \mathrm{mg} / 50 \mathrm{mg}$ per $\mathrm{kg}$ body weight, intraperitoneally (i.p.)), and processed as we have described previously [36]. Immunostaining was performed on the right hemisphere of coronal fixed brain sections $(40 \mu \mathrm{m})$ with mouse-monoclonal tau antibodies that stain pathological tau, PHF-1(1:1000), against the PSer396, 404 epitope and MC1 (1:100) which recognizes a conformational epitope. Both antibodies were generously provided by Peter Davies (Feinstein Institute for Medical Research, Manhasset, NY).

\section{Cultures}

Performed as previously described [10]. 


\section{Immunoblotting \\ Animals}

The left hemisphere of the brain was homogenized in (5x vol/w) modified RIPA buffer as described [24]. The brain homogenate was centrifuged $(20,000 \mathrm{xg})$ for $20 \mathrm{~min}$ at $20{ }^{\circ} \mathrm{C}$ and supernatants collected as LSS (Low Speed Supernatant). After protein quantification, the volumes were adjusted for equal protein concentration with dilution in modified $\mathrm{O}+$ buffer as described [24], boiled for $5 \mathrm{~min}$, and loaded onto $12 \%$ polyacrylamide gel. For the sarkosyl insoluble fraction, $10 \%$ sarkosyl solution was added to LSS, and the sample mixed for $30 \mathrm{~min}$ at room temperature, then centrifuged at $100,000 \mathrm{xg}$ for $1 \mathrm{~h}$ at $20{ }^{\circ} \mathrm{C}$. The pellet was then washed in $1 \%$ sarkosyl solution and spun again for $100,000 \mathrm{xg}$ for $1 \mathrm{~h}$ at $20{ }^{\circ} \mathrm{C}$. It was then air dried for $30 \mathrm{~min}$, mixed with $50 \mu \mathrm{l}$ of modified $\mathrm{O}+$ buffer, vortexed for $1 \mathrm{~min}$, then boiled for $5 \mathrm{~min}$ and denoted the sarkosyl pellet (SP) fraction. The LSS and sarkosyl insoluble fractions were both incubated with CP27. The LSS fraction was also probed for total tau using Tau-5, phospho-tau using PHF-1 and oligomeric tau using T22 [37]. All soluble tau blots were normalized using GAPDH.

\section{Cultures}

Performed as described previously [10]. Prior to immunoblotting, all samples from control and treated cells were assayed for total protein concentration, and normalized accordingly. Neuronal cultures were probed with antibodies recognizing neuronal marker NeuN, total tau (Dako) and tau phosphorylated at Ser199 (SantaCruz). NeuN was used to measure PHF induced cytotoxicity [38-43] and to control for tau levels [44]. Because the expression of tau protein can vary between animals, even when on a homozygous background, each experiment was preformed using cells prepared from a single animal. Untreated control cells for each experiment were also prepared using the same animal. Thus, each experiment has its own unique set of control samples.

\section{Behavioral studies \\ Sensorimotor tests}

Rotarod This test is used to measure forelimb and hindlimb motor coordination and balance. This procedure was designed to assess motor behavior without a practice confound. It was performed similar to as previously described in detail [30].

Locomotor activity This test was performed similarly to those previously described in detail [30].

\section{Cognitive tests \\ Closed field symmetrical maze}

This apparatus is a rectangular field, $65 \mathrm{~cm}$ square with $10 \mathrm{~cm}$ high walls divided into 36 squares. Two boxes, $(16 \times 23 \times 10 \mathrm{~cm})$, are attached to the square at its diagonal corners. The symmetrical maze is a modification of the Hebb-Williams and Rabinovitch-Rosvold tests as we have described [30, 45, 46]. Briefly, each end box functions as both a start box and a goal box. The mice run in opposite directions on alternate trials, thereby eliminating intertrial handling which should minimize stress. The barriers are placed in the field in symmetrical patterns, so that mice face the same turns going in either direction within a given problem. On day 0 , mice were adapted to a water-restriction schedule $(2 \mathrm{~h}$ daily access to water) and habituated in the same environment as used for testing. On day 1, all mice were given saccharine-flavored water, for $10 \mathrm{~min}$ in each box. On day 2 , they were placed in the start box and permitted to explore the field and enter the goal box, in which the saccharine-water reward $(0.05 \mathrm{ml})$ was available. The door to each box was manually opened and closed to allow entry and exit. When the mice were running reliably from the start box to the goal box, they were given four practice trials under the same condition. On day 3 , they were given one practice session on a simple problem, in which two barriers were placed in different symmetrical positions in the field so as to obstruct direct access to the goal box. This practice test was repeated for 4 trials. On day 4, formal testing consisted of three barriers graded for the most difficulty (Maze 7), based on our prior findings $[30,45,46]$. Mice were given five trials with an intertrial interval of $2 \mathrm{~min}$. Performance was scored manually by the same observer, who was blinded to the treatment received, in terms of errors (ie, entries and reentries into designated error zones) and time to complete each trial.

In the acute treatment study, mice were then split into control and treatment group, which had similar average tests scores and group variance, taking into account as well their performance on the sensorimotor tests. The mice were retested without practice period after the treatment period.

\section{Fear-conditioning}

This test was performed as described previously [24]. On the training day, mice were allowed to explore for $2 \mathrm{~min}$ in the test chamber. The conditioned stimulus (CS; a white noise $80 \mathrm{~dB}$ sound) was presented for $30 \mathrm{~s}$ and followed immediately by a mild foot shock (2 s, $0.5 \mathrm{~mA}$ ) that served as the unconditioned stimulus (US). After 2 min, the mice received a second CS-US pairing. The Freeze Frame monitor system (San Diego Instruments) was used to control the timing of CS and US 
presentations and to record freezing behavior. During the conditioning procedure, response to the foot shock -typically run, jump, or vocalize- were also recorded. Mice were tested for contextual fear in $3 \mathrm{~h}$ for short term memory and $24 \mathrm{~h}$ for long term memory, during which mice were placed into the original test chamber for $5 \mathrm{~min}$ in the absence of CS and freezing behavior was recorded.

\section{Statistics}

All data were analyzed with GraphPad Prism 6 (San Diego, CA).

\section{Ex vivo culture}

In the competitive ELISA assay, data from the group that did not receive PHF was used to represent $100 \%$ of the normal binding to the plate, with the other conditions normalized to these values. Values were fitted to a nonlinear curve competitive binding formula to determine the IC50 for each antibody. LDH data were analyzed using a one-way AVOVA. Data from PHF and antibody time courses were normalized using untreated cells from the same animal. For each individual time point, the percentage of the control for each sample was determined, and the average and standard error of the mean calculated. For each set of experiments a two-way ANOVA was used to determine the effect of individual treatment group and time. For tau spreading experiments, the percentage of PHF positive cells in each image was determined, and used to determine the average and standard error of the mean for each group. Treatment groups were then compared using a one-way ANOVA. Significant differences between individual time points, and/or groups, were determined using Tukey's multiple comparisons test.

\section{In vivo}

The behavioral data with pre- and post-testing (CFSM, Rotorod and Open Field) was analyzed by a paired $t$ test. The Fear Conditioning data and tau levels on western blots were analyzed with an unpaired $t$-test. Two tailed $\mathrm{t}$-tests were used to compare immunoblot results. Welch correction was used if the data failed a test of equal variance. When data failed two out of three normality tests (KS, D'Agostino \& Pearson omnibus, and Shapiro-Wilk normality tests) non-parametric MannWhitney test was used. The effects of gender and antibody treatment on behavior and immunoblot results were further assessed using a two-way ANOVA.

\section{Additional file}

Additional file 1: Figure S1. htau mice show limited tau pathology which is not affected by 4E6 treatment. (PDF 79 kb)

\section{Abbreviations}

$\mathrm{Ab}$, antibody; ANOVA, analysis of variance; CFSM, Closed field symmetrical maze; DC, dansyl cadaverine; ELISA, enzyme linked immunosorbent assay; IC50: half maximal inhibitory concentration; IgG, immunoglobulin $G ; K_{D}$, dissociation constant; LDH, lactate dehydrogenase; PHF, paired helical filament; Tg, transgenic; WT, wild type

\section{Acknowledgments}

This work was supported by the National Institutes of Health (NIH Grants NS077239, AG032611, and AG020197 -EMS), the Alzheimer's Association (2016NIRG-397228, EEC), and the Blas Frangione Foundation (EEC) as well as by Sandy NIH grants 1R24OD018340 and 1R24OD018339. We thank Dr. Peter Davies (Albert Einstein College of Medicine and Long Island Jewish Medical Center) for the tau antibodies PHF1, MC1 and CP27, and Dr. Edwin VasquezCintron (New York University School of Medicine) for the botulinum toxin. We acknowledge the use of tissues procured by the National Disease Research Interchange (NDRI) with support from NIH grant 2 U42 OD011158.

\section{Authors' contributions}

EMS designed the overall approach for the in vivo experiments, and the culture studies with EEC. EMS was also involved in data analysis for both in vivo and cultures studies and in writing the manuscript. EEC was involved in the design, performed and analyzed the data from the culture experiments, and wrote the relevant sections of the manuscript. YL performed the in vivo experiments, data analysis and participated in writing the relevant portions of the manuscript. DBS assisted in PHF extraction and in performing the cell culture experiments. HBR, WJR, VG, and JG assisted in performing the in vivo studies, as well as related tissue and data analysis. SR prepared the human tau fractions. SK performed the Biacore assay. $\mathrm{CH}$ designed and conducted the fear conditioning experiments with $J \mathrm{~L}$. All authors read and approved the final draft.

\section{Competing interests}

EMS is an inventor on patents on tau immunotherapies and related diagnostics which are assigned to New York University and licensed to $\mathrm{H}$. Lundbeck A/S. NYU technology on tau immunotherapy is licensed to and is being codeveloped with $\mathrm{H}$. Lundbeck A/S. The other authors have no competing interests.

\section{Ethical approval}

Under US ethical guidelines, this study did not need ethics approval concerning use of the human tissue.

\section{Author details}

${ }^{1}$ Departments of Neuroscience and Physiology, New York University School of Medicine, Medical Science Building, MSB459, 550 First Avenue, New York, NY 10016, USA. ${ }^{2}$ Department of Integrative Physiology, Institute for Behavioral Genetics, University of Colorado, Boulder, CO 80309, USA. ${ }^{3}$ Departments of Psychiatry, New York University School of Medicine, New York, NY 10016, USA.

Received: 30 March 2016 Accepted: 4 August 2016

Published online: 30 August 2016

\section{References}

1. Asuni AA, et al. Immunotherapy targeting pathological tau conformers in a tangle mouse model reduces brain pathology with associated functional improvements. J Neurosci. 2007;27(34):9115-29.

2. Sigurdsson EM. Immunotherapy targeting pathological tau protein in Alzheimer's disease and related tauopathies. J Alzheimers Dis. 2008;15(2): 157-68.

3. Sigurdsson EM, Wisniewski T, Frangione B. Infectivity of amyloid diseases. Trends Mol Med. 2002;8(9):411-3.

4. Congdon EE, Krishnaswamy S, Sigurdsson EM. Harnessing the immune system for treatment and detection of tau pathology. J Alzheimers Dis. 2014;40 Suppl 1:S113-21

5. Pedersen JT, Sigurdsson EM. Tau immunotherapy for Alzheimer's disease. Trends Mol Med. 2015;21(6):394-402.

6. Goedert $M$, et al. Prion-like mechanisms in the pathogenesis of tauopathies and synucleinopathies. Curr Neurol Neurosci Rep. 2014;14(11):495. 
7. Walker $L C$, et al. Mechanisms of protein seeding in neurodegenerative diseases. JAMA Neurol. 2013;70(3):304-10.

8. Nelson PT, et al. Correlation of Alzheimer disease neuropathologic changes with cognitive status: a review of the literature. J Neuropathol Exp Neurol. 2012;71(5):362-81.

9. Collin L, et al. Neuronal uptake of tau/pS422 antibody and reduced progression of tau pathology in a mouse model of Alzheimer's disease. Brain. 2014;137(Pt 10):2834-46.

10. Congdon EE, et al. Antibody uptake into neurons occurs primarily via clathrin-dependent Fcgamma receptor endocytosis and is a prerequisite for acute tau protein clearance. J Biol Chem. 2013;288(49):35452-65.

11. Gu J, Congdon EE, Sigurdsson EM. Two novel Tau antibodies targeting the 396/404 region are primarily taken up by neurons and reduce Tau protein pathology. J Biol Chem. 2013;288(46):33081-95.

12. Kondo A, et al. Antibody against early driver of neurodegeneration cis P-tau blocks brain injury and tauopathy. Nature. 2015;523(7561):431-6.

13. Krishnamurthy PK, Deng Y, Sigurdsson EM. Mechanistic studies of antibodymediated clearance of Tau aggregates using an ex vivo brain slice model. Front Psychiatry. 2011;2:59.

14. Krishnaswamy $\mathrm{S}$, et al. Antibody-derived in vivo imaging of tau pathology. J Neurosci. 2014:34(50):16835-50.

15. Castillo-Carranza DL, et al. Passive immunization with Tau oligomer monoclonal antibody reverses tauopathy phenotypes without affecting hyperphosphorylated neurofibrillary tangles. J Neurosci. 2014;34(12):4260-72.

16. d'Abramo C, et al. Tau passive immunotherapy in mutant P301L mice: antibody affinity versus specificity. PLoS One. 2013;8(4), e62402.

17. Yanamandra $\mathrm{K}$, et al. Anti-tau antibodies that block tau aggregate seeding in vitro markedly decrease pathology and improve cognition in vivo. Neuron. 2013;80(2):402-14.

18. Bumbaca $D$, et al. Physiochemical and biochemical factors influencing the pharmacokinetics of antibody therapeutics. AAPS J. 2012;14(3):554-8.

19. Igawa $T$, et al. Reduced elimination of $\lg G$ antibodies by engineering the variable region. Protein Eng Des Sel. 2010;23(5):385-92.

20. Andorfer $C$, et al. Hyperphosphorylation and aggregation of tau in mice expressing normal human tau isoforms. J Neurochem. 2003;86(3):582-90.

21. Davis J, et al. Lost in transgenesis: a user's guide for genetically manipulating the mouse in cardiac research. Circ Res. 2012;111(6):761-77.

22. Henriques A, Pitzer C, Schneider A. Characterization of a novel SOD-1(G93A) transgenic mouse line with very decelerated disease development. PLoS One. 2010;5(11), e15445.

23. Lee VM, Wang J, Trojanowski JQ. Purification of paired helical filament tau and normal tau from human brain tissue. Methods Enzymol. 1999;309:81-9.

24. Levenga J, et al. Tau pathology induces loss of GABAergic interneurons leading to altered synaptic plasticity and behavioral impairments. Acta Neuropathol Commun. 2013;1:34.

25. Fa M, Puzzo D, Piacentini R, Staniszewski A, Zhang H, Baltrons MA, et al, Extracellular tau oligomers produce an immediate impairment of LTP and memory. Sci Rep. 2016;6:19393.

26. Funk KE, et al. Distinct therapeutic mechanisms of Tau antibodies: promoting microglial clearence versus blocking neuronal uptake. J Biol Chem. 2015;290(35):21652-62.

27. Luo W, et al. Microglial internalization and degradation of pathological tau is enhanced by an anti-tau monoclonal antibody. Sci Rep. 2015;5:11161.

28. Masliah $\mathrm{E}$, et al. Passive immunization reduces behavioral and neuropathological deficits in an alpha-synuclein transgenic model of Lewy body disease. PLoS One. 2011;6(4), e19338.

29. Lewis J, et al. Neurofibrillary tangles, amyotrophy and progressive motor disturbance in mice expressing mutant (P301L) tau protein. Nat Genet. 2000;25(4):402-5.

30. Boutajangout $\mathrm{A}$, et al. Cognitive and sensorimotor tasks for assessing functional impairments in mouse models of Alzheimer's disease and related disorders. Methods Mol Biol. 2012;849:529-40.

31. Chang E, et al. Pathogenic missense MAPT mutations differentially modulate tau aggregation propensity at nucleation and extension steps. J Neurochem. 2008;107(4):1113-23.

32. Congdon EE, et al. Nucleation-dependent tau filament formation: the importance of dimerization and an estimation of elementary rate constants. J Biol Chem. 2008;283(20):13806-16.

33. Shammas SL, et al. A mechanistic model of tau amyloid aggregation based on direct observation of oligomers. Nat Commun. 2015;6:7025.
34. Congdon EE, et al. Methylthioninium chloride (methylene blue) induces autophagy and attenuates tauopathy in vitro and in vivo. Autophagy. 2012;8(4):609-22.

35. Pecho-Vrieseling $E$, et al. Transneuronal propagation of mutant huntingtin contributes to non-cell autonomous pathology in neurons. Nat Neurosci. 2014;17(8):1064-72.

36. Rajamohamedsait HB, Sigurdsson EM. Histological staining of amyloid and pre-amyloid peptides and proteins in mouse tissue. Methods Mol Biol. 2012;849:411-24.

37. Lasagna-Reeves CA, et al. Alzheimer brain-derived tau oligomers propagate pathology from endogenous tau. Sci Rep. 2012;2:700.

38. Murphy KE, et al. Reduced glucocerebrosidase is associated with increased alpha-synuclein in sporadic Parkinson's disease. Brain. 2014;137(Pt 3):834-48.

39. Park S, Jung Y. Combined actions of Na/K-ATPase, NCX1 and glutamate dependent NMDA receptors in ischemic rat brain penumbra. Anat Cell Biol. 2010;43(3):201-10.

40. McKiernan RC, et al. Reduced mature microRNA levels in association with dicer loss in human temporal lobe epilepsy with hippocampal sclerosis. PLoS One. 2012;7(5), e35921.

41. Karuppagounder SS, et al. Changes in inflammatory processes associated with selective vulnerability following mild impairment of oxidative metabolism. Neurobiol Dis. 2007;26(2):353-62.

42. Lesuisse C, Martin L. Long-term culture of mouse cortical neurons as a model for neuronal development, aging, and death. J Neurobiol. 2002;51(1):9-23.

43. Ryu HW, Park CW, Ryu KY. Disruption of polyubiquitin gene Ubb causes dysregulation of neural stem cell differentiation with premature gliogenesis. Sci Rep. 2014;4:7026

44. Jin N, et al. Truncation and activation of GSK-3beta by calpain I: a molecular mechanism links to tau hyperphosphorylation in Alzheimer's disease. Sci Rep. 2015;5:8187.

45. Asuni AA, et al. Vaccination of Alzheimer's model mice with Abeta derivative in alum adjuvant reduces Abeta burden without microhemorrhages. Eur J Neurosci. 2006:24(9):2530-42.

46. Boutajangout A, Quartermain D, Sigurdsson EM. Immunotherapy targeting pathological tau prevents cognitive decline in a new tangle mouse model. J Neurosci. 2010;30(49):16559-66.

\section{Submit your next manuscript to BioMed Central and we will help you at every step:}

- We accept pre-submission inquiries

- Our selector tool helps you to find the most relevant journal

- We provide round the clock customer support

- Convenient online submission

- Thorough peer review

- Inclusion in PubMed and all major indexing services

- Maximum visibility for your research

Submit your manuscript at www.biomedcentral.com/submit 